MIT-CTP\#2357

hep-ph/9412246

INFNCA-TH-94-16

\title{
Constraining Spectral Functions at Finite Temperature and Chemical Potential with Exact Sum Rules in Asymptotically Free Theories
}

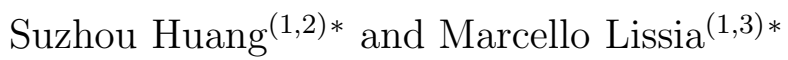 \\ (1) Center for Theoretical Physics, Laboratory for Nuclear Science and Department of Physics, \\ Massachusetts Institute of Technology, Cambridge, Massachusetts 02139 \\ ${ }^{(2)}$ Department of Physics, FM-15, University of Washington, Seattle, Washington 98195 \\ (3) Istituto Nazionale di Fisica Nucleare, via Ada Negri 18, I-09127 Cagliari, Italy \\ and Dipartimento di Fisica dell'Università di Cagliari, I-09124 Cagliari, Italy
}

(September 3, 2018)

\begin{abstract}
Within the framework of the operator product expansion (OPE) and the renormalization group equation (RGE), we show that the temperature and chemical potential dependence of the zeroth moment of a spectral function (SF) is completely determined by the one-loop structure in an asymptotically free theory, and in particular in QCD. Logarithmic corrections are found to play an essential role in the derivation. This exact result constrains the shape of SF's, and implies striking effects near phase transitions. Phenomenological parameterizations of the SF, often used in applications such as the analysis of lattice QCD data or QCD sum rule calculations at finite temperature and baryon density must satisfy these constraints. We also explicitly illustrate in detail the exact sum rule in the Gross-Neveu model.
\end{abstract}




\section{INTRODUCTION}

Hadronic properties at finite temperature and baryon density are of great importance in the phenomenology of heavy ions collisions, star interior and the early universe. Moreover, the theoretical expectation of transitions to a chirally symmetric phase and, perhaps, to a quark-gluon plasma phase contributes to the interest in studying the effect of matter and temperature on the quantum chromodynamics (QCD) vacuum.

Our present understanding of QCD at finite temperature $(T)$ and baryon density (or chemical potential $\mu$ ) is mainly limited in the Euclidean realm, due to the lack of nonperturbative and systematic calculating tools directly in the Minkowski space. Typical methods, with QCD Lagrangian as the starting point, are the OPE and lattice simulations. Because these two formulations are intrinsically Euclidean, only static quantities are conveniently studied. In order to gain dynamical informations, which are more accessible experimentally, the analytic structure implemented through dispersion relations often have to be invoked within the theory of linear response.

In principle, dispersion relations allow the determination of the spectral function (SF), which carries all the real-time information, from the corresponding correlator in the Euclidean region. In practice, realistic calculations, e.g. OPE or lattice simulations, yield only partial information on the correlators, making impossible a direct inversion of the dispersion relation.

Therefore, the standard approach assumes a phenomenological motivated functional form with several parameters for the SF, and uses the information from the approximate correlator, mediated by the dispersion relation, only to determine the value of parameters by a fit. This approach has been quite successful at zero temperature and density, thanks to the fortuitous situation that we roughly know how to parameterize the SF's in many cases. Two important examples are the QCD sum rules pioneered by Shifman, Vainshtein and Zakharov [1,2], and the analysis of lattice QCD data [3]. So far, standard parameterizations have included poles plus perturbative continuum [4].

The success of such approaches heavily rests on our good understanding of the qualitative behavior of SF's at zero $(T, \mu)$. We can find other such favorable examples in the low- $T$ regime [5], where the shape of the $\mathrm{SF}$ has the same qualitatively features of the zero $(T, \mu)$ case, or even in the high- $T$ regime for simple models [6], for which the functional form of the $\mathrm{SF}$ is known.

The QCD sum rules approach has been extended also to systems at finite temperature [7,8,5]. The lack of experimental data, and of reliable nonperturbative calculations has prompted people to use the same kind of parameterizations that have worked so well at zero temperature with, at most, perturbative corrections. We believe that physical results at finite $(T, \mu)$ can be strongly biased by this assumption. In fact, recent interpretations of lattice simulation data [9-11] appear to indicate the existence of such problems.

The purpose of this work is to derive exact sum rules that constrain the variation of SF's with $(T, \mu)$. In addition, we apply these sum rules to the chiral phase transition, and demonstrate that SF's in some channels are drastically modified compared to both their zero $(T, \mu)$ and their perturbative shapes. This result confirm our worries about non-trivial effect of finite $T$ or baryon density on the shape of the SF.

Our derivation of these exact sum rules, based on the OPE and the RGE, has a closer 
relation with the derivation of sum rules for deep inelastic scatterings than with the QCD sum rule approach of SVZ [1]. In fact, we establish relationships between moments of the $\mathrm{SF}$ and corresponding condensates as functions of $(T, \mu)$, without assuming any functional form of the SF.

In the derivation process, we find that the logarithmic corrections are essential to establish the exact sum rules. In contrast, the QCD logarithmic corrections are only marginally relevant in the finite energy sum rules, and hence are rarely discussed in the literature. To properly take into account the logarithmic corrections, a repeated partial integration method is used to match the relevant asymptotic expansions.

Since no further assumptions other than the validity of the OPE and RGE are involved in the derivation, our sum rules are very general and can be applied anywhere in the $(T, \mu)$ plane, even near or at the boundary of a phase transition.

The paper is organized as follows. In section II we present the general derivation of exact sum rules in asymptotically free theories. The matching of asymptotic behaviors of the correlator and the dispersion integral, including explicitly their logarithmic corrections, are carefully discussed. In section III we illustrate each single step of the derivation in a soluble model, the Gross-Neveu model in the large- $N$ limit. In this model we can calculate exactly all the relevant quantities (spectral functions, Wilson coefficients, condensates, anomalous dimensions and correlators in space-like region, etc.), and, therefore, give a concrete example of how our method works. The application of our method to the derivation of exact sum rules for the mesonic channels in QCD is presented in section IV. In the same section, we also discuss the phenomenological consequences of the exact sum rules near chiral restoration phase transitions. Finally, in section $\mathrm{V}$ we summarize our work, draw some conclusions, and discuss possible future directions.

\section{GENERAL DERIVATION}

We start this section with a short review of the linear response theory, the OPE and the RGE. Next we introduce a convenient subtraction in the dispersion relation for studying the dependence of SF's on $T$ and $\mu$.

Then, we present in detail a crucial part of our method: how to match the asymptotic OPE expansion with a corresponding asymptotic expansion of the SF and its dispersion integral. This approach is necessary for properly taking into account the logarithmic corrections, and studying the convergence properties of the relevant moments of the SF. More naive approaches not only yield, in general, incorrect sum rules, but might also fail to recognize that a given sum rule does not exist in the first place, since the integral of the SF involved is divergent.

Finally, a comparison of the two asymptotic expansions leads to the desired exact sum

rules. We end this section with some general comments on the derivation and meaning of these sum rules. 


\section{A. Linear Response}

The real-time linear response [12] to an external source, $S(x)$, coupled to a renormalized current $J(x)$ in the form of $\Delta \mathcal{L}=S(x) J(x)$ is given by the retarded correlator:

$$
K(x ; T, \mu) \equiv \theta\left(x_{0}\right)\langle[J(x), J(0)]\rangle_{T, \mu},
$$

where the average is on the grand canonical ensemble specified by temperature $T$ and chemical potential $\mu$. Disregarding possible subtraction terms, which are $(T, \mu)$-independent since they are related to short distance properties of the theory, we can write the following dispersion relation for the frequency dependence of the retarded correlation function:

$$
\tilde{K}(\omega, \boldsymbol{k} ; T, \mu)=\int_{0}^{\infty} d u^{2} \frac{\rho(u, \boldsymbol{k} ; T, \mu)}{u^{2}-(\omega+i \epsilon)^{2}} .
$$

For convenience, we discuss only the uniform limit $(\boldsymbol{k}=0)$ and, from now on, drop the momentum label. We wish to emphasize that, even if the derivation of the sum rules requires the analytic continuation of Eq. (2) into the Euclidean region $(\omega \rightarrow i Q)$, the dispersion integral is, nevertheless, determined by the singularities of $\rho(u ; T, \mu)$ in the Minkowski region. One should keep clearly in mind the distinction between these real-time singularities with the ones related to screening phenomena.

\section{B. The OPE and the RGE}

In asymptotically free theories, the correlator $\tilde{K}(\omega=i Q ; T, \mu)$ is calculable in terms of the OPE [13] asymptotic series in the limit of $Q^{2} \rightarrow \infty$. For a given renormalization prescription, the asymptotic expansion reads

$$
\tilde{K}(i Q, \kappa ; T, \mu) \sim \tilde{K}_{0}(i Q, \kappa)+\sum_{n} C_{n}\left(Q^{2}, m^{2}(\kappa), g^{2}(\kappa), \kappa\right)\left\langle\left[O_{n}\right]_{\kappa}\right\rangle_{T, \mu}
$$

where $m(\kappa), g(\kappa)$, the $\left[O_{n}\right]_{\kappa}$ 's and the $C_{n}$ 's are, respectively, the current quark mass, the coupling constant, the renormalized composite operators, and their corresponding Wilson coefficients at the subtraction scale $\kappa$. Here and in the following, we use the symbol $\sim$ to denote asymptotic equality. We explicitly singled out the perturbative term $\tilde{K}_{0}(i Q, \kappa)$, which corresponds to the identity operator and, being independent of $T$ and $\mu$, is calculated at $(T, \mu)=0$. This term is the only one that is not suppressed by powers of $1 / Q^{2}$ and can depend on an overall subtraction scale, which we identify, for simplicity, with the same subtraction scale $\kappa$ used in the renormalization of the operators. It is important to note that the information of the ensemble average is encoded in the matrix elements of composite operators, while the Wilson coefficients, including $\tilde{K}_{0}$, are independent of $T$ and $\mu$. Although the matrix elements $\left\langle\left[O_{n}\right]_{\kappa}\right\rangle_{T, \mu}$ cannot be determined perturbatively, the $Q^{2}$-dependence of the Wilson coefficient $C_{n}$ is controlled by the following renormalization group equation [13]

$$
\left[\kappa \frac{d}{d \kappa}+2 \Gamma_{J}-\Gamma_{n}\right] C_{n}\left(Q^{2}, m^{2}(\kappa), g^{2}(\kappa), \kappa\right)=0
$$


where

$$
\kappa \frac{d}{d \kappa}=\kappa \frac{\partial}{\partial \kappa}+\beta \frac{\partial}{\partial g}-\Gamma_{m} \frac{\partial}{\partial m},
$$

and $\Gamma_{J}, \Gamma_{n}$ and $\Gamma_{m}$ are the anomalous dimensions for the current $J$, the operator $O_{n}$ and the current quark mass $m$ respectively. For the purpose of illustration, we are only considering operators that do not mix under renormalization, but the mixing will be properly taken into account when necessary. The standard approach [13] to the renormalization group equation is the introduction of a running coupling $g^{2}(Q)$ and a running mass $m(Q)$. In asymptotically free theories $g^{2}(Q)$ vanishes logarithmically at large $Q^{2}$. It is therefore meaningful to consider, in this limit, a perturbative expansion of the renormalization group equation functions

$$
\Gamma_{i}=-\gamma_{i} g^{2}+\mathcal{O}\left(g^{4}\right), \text { and } \beta=-b g^{4}+\mathcal{O}\left(g^{6}\right),
$$

where $i=J, n, m$, while $\gamma_{i}, b$ are pure numbers determined by a one-loop calculation. Within this perturbative context, Eq. (14) can be solved [13]:

$$
C_{n}\left(Q^{2}, m(\kappa), g^{2}(\kappa), \kappa\right)=\left(\frac{1}{Q^{2}}\right)^{\frac{d_{n}}{2}}\left(\frac{g^{2}(Q)}{g^{2}(\kappa)}\right)^{\frac{2 \gamma_{J}-\gamma_{n}}{2 b}} \times c_{n}\left(\frac{m^{2}(Q)}{Q^{2}}, g^{2}(Q)\right),
$$

where $d_{n}$ is the canonical dimension of the composite operator $O_{n}$ minus the dimension of $\tilde{K}$ in units of a mass, and the $c_{n}\left(m^{2}(Q) / Q^{2}, g^{2}(Q)\right)$ 's are calculable perturbatively. Corrections to this result coming from nonleading powers of $g^{2}$ in the expansions of the renormalization group equation functions, see Eq. (5), are analytic in $g^{2}(Q)=1 /\left(b \ln Q^{2}\right)$ and hence can be absorbed into $c_{n}$. Therefore, the leading term in the large- $Q^{2}$ limit is totally characterized by the one-loop structure of the theory.

\section{Subtraction and $(T, \mu)$-dependence}

To study the temperature and chemical potential dependence of $\tilde{K}$, we only need to consider the difference $\Delta \tilde{K}(i Q)$ :

$$
\Delta \tilde{K}(i Q) \equiv \tilde{K}(i Q, \kappa ; T, \mu)-\tilde{K}\left(i Q, \kappa ; T^{\prime}, \mu^{\prime}\right)=\int_{0}^{\infty} d u^{2} \frac{\Delta \rho(u)}{u^{2}+Q^{2}},
$$

where $\Delta \rho(u) \equiv \rho(u ; T, \mu)-\rho\left(u ; T^{\prime}, \mu^{\prime}\right)$. This subtraction is crucial to remove $\tilde{K}_{0}(i Q, \kappa)$, which contains terms not suppressed by powers of $1 / Q^{2}$ and is explicitly dependent on the renormalization point. The OPE asymptotic expansion of $\Delta \tilde{K}(i Q)$ is then

$$
\Delta \tilde{K}(i Q) \sim \sum_{n}\left(\frac{1}{Q^{2}}\right)^{\frac{d_{n}}{2}}\left(\frac{g^{2}(Q)}{g^{2}(\kappa)}\right)^{\frac{2 \gamma_{J}-\gamma_{n}}{2 b}} \times c_{n}\left(\frac{m^{2}(Q)}{Q^{2}}, g^{2}(Q)\right) \Delta\left\langle\left[O_{n}\right]_{\kappa}\right\rangle
$$

where $\Delta\left\langle\left[O_{n}\right]_{\kappa}\right\rangle$ denotes the difference of the expectation values of $\left[O_{n}\right]_{\kappa}$ in the ensembles specified by $(T, \mu)$ and $\left(T^{\prime}, \mu^{\prime}\right)$, respectively. Since the current quark mass only runs logarithmically in asymptotically free theories, we can safely ignore the corrections to the $c_{n}$ 's due to their dependence on $m^{2}(Q) / Q^{2}$ when in deep Euclidean limit. Notice that the subtraction has made $\Delta \tilde{K}(i Q)$ independent of the renormalization point $\kappa$, i.e. $\Delta \tilde{K}(i Q)$ satisfies a homogeneous RGE. 


\section{Asymptotic Expansion}

Since $c_{n}\left(m^{2}(Q) / Q^{2}, g^{2}(Q)\right)$ is perturbative and hence can be expanded in power of $g^{2}(Q)$ (and denoting the first non-vanishing power as $\left.\nu_{n}^{(0)}\right)$, the left-hand side of Eq. (7) can be expressed as a double (in $1 / Q^{2}$ and $g^{2}(Q)$ ) asymptotic expansion of the form:

$$
\Delta \tilde{K}(i Q) \sim \sum_{n, \nu=0}^{\infty} \frac{c_{n}^{(\nu)}(\kappa) \Delta\left\langle\left[O_{n}\right]_{\kappa}\right\rangle}{Q^{d_{n}}}\left[g^{2}(Q)\right]^{\nu+\eta_{n}},
$$

where the exponents $\eta_{n}=\nu_{n}^{(0)}+\left(2 \gamma_{J}-\gamma_{n}\right) /(2 b)$ and the $Q^{2}$-independent coefficients $c_{n}^{(\nu)}(\kappa)$ are again known perturbatively. It is important to emphasize here that the leading term in $g^{2}(Q)$ for a given power of $1 / Q^{2}$ is controlled by the relevant anomalous dimensions.

We proceed by making an analogous asymptotic expansion of $\Delta \rho(u)$ valid for $u^{2} \in$ $\left(\lambda^{2}, \infty\right)$ with $\lambda^{2}$ suitably large but otherwise arbitrary:

$$
\Delta \rho(u) \equiv \Delta \rho_{\text {power }}(u)+\Delta \rho_{\exp }(u)
$$

with

$$
\Delta \rho_{\text {power }}(u) \sim \sum_{n=0}^{\infty} \frac{\left[\ln \left(u^{2}\right)\right]^{-\xi_{n}}}{u^{2(n+1)}} \sum_{\nu=0}^{\infty} a_{n}^{(\nu)}(T, \mu)\left[\ln \left(u^{2}\right)\right]^{-\nu},
$$

where we have explicitly isolated in $\Delta \rho_{\exp }(u)$ all terms that vanish exponentially when $u^{2} \rightarrow \infty$, such as the pole contributions to $\Delta \rho(u)$ or terms containing the factor $\exp (-u / T)$. To simplify the notation we have chosen the units such that the running coupling has the form at one-loop level $g^{2}(u)=1 /\left(b \ln u^{2}\right.$ ) (or equivalently, energy scales are measured in units of the relevant $\Lambda$-parameter).

This ansatz is sufficient to produce an asymptotic series of the form of Eq. (91) to oneloop level. More generally, one could replace $1 / \ln u^{2}$ with the full running coupling $g^{2}(u)$ in the asymptotic sequence and generalize the method we are going to describe; the general strategy involved in this generalization can be found, for example, in Ref. [14]. Since we are presently only interested in one-loop calculations, we can regard $g^{2}(Q)$ and $1 / \ln Q^{2}$ to be proportional. It is easy to recognize that the sum over " $n$ " is meant to match the sum over mass dimension in Eq. (9), while the sum over " $\nu$ " will match the sum over the order in perturbation series. The existence of anomalous dimensions in the OPE makes it necessary to introduce $\xi_{n}$ in the expansion for the spectral function. In the following we ignore the dependence of $\xi_{n}$ on " $n$ ", as we have already ignored the fact that there generally exist more than one operator at a given dimension, and write $\xi$ to avoid a too cumbersome notation. The complete notation will be restored when necessary.

Before we proceed further, we wish to discuss whether it is possible that additional terms might appear in the expansion of $\Delta \rho_{\text {power }}(u)$ in Eq. (10b). In general, our asymptotic expansion procedure is powerful enough to exclude this possibility within the framework of the OPE. In fact, it allows to verify unambiguously that terms different from the ones already present generate, when substituted in the dispersion integral, terms that are missing in the OPE. Let us examine two specific examples that might be suspected to exist otherwise. First, dimensional arguments could suggest terms like $g^{2} T / \sqrt{u^{2}}$. Our procedure shows that any 
$1 / \sqrt{u^{2}}$ term in $\Delta \rho(u)$ would generate a $1 / \sqrt{Q^{2}}$ term in $\Delta \tilde{K}(i Q)$, which does not correspond to any known condensate, and it is therefore excluded. The second example is given by terms such as $\ln \left(T^{2} / u^{2}\right)$, which are naturally produced by elementary perturbative calculations of the spectral function: are such terms present in Eq. (10b)? It is indeed true that such terms appear in the high- $T$ expansion of the spectral function at fixed $u^{2}$. However, the expansion of the spectral function that is relevant for comparing to the OPE is a high- $u^{2}$ expansion at fixed $T$. In the next section an explicit calculation in the Gross-Neveu model will illustrate the general fact that, contrary to the high- $T$ expansion, the high- $u^{2}$ expansion does not generate terms like $\ln \left(T^{2} / u^{2}\right)$.

At this stage, we can already recognize a fundamental, and often overlooked, characteristic of the spectral function. If we insert the term $\Delta \rho_{\exp }(u)$ in the dispersion integral, we only obtain pure powers of $1 / Q^{2}$, since the exponential convergence allows a naive expansion of the factor $1 /\left(u^{2}+Q^{2}\right)$ (Watson's Lemma [15]). Therefore logarithmic corrections, i.e. powers of $g^{2}(Q)$, come solely from the $\Delta \rho_{\text {power }}(u)$ term. Because we know that the running coupling $g^{2}(Q)$ is always present in the OPE series, the term $\Delta \rho_{\text {power }}(u)$ must be present in the subtracted $\mathrm{SF}$, and it obviously dominates the asymptotic regime $\left(u^{2} \rightarrow \infty\right)$. This fact immediately implies that only a finite number of moments of the subtracted SF can possibly be finite, i.e. the naive expansion of the factor $1 /\left(u^{2}+Q^{2}\right)$ is generally wrong, and that logarithmic corrections play a important role.

A standard method to tackle the dispersion integral in the large- $Q^{2}$ limit is the Mellin transform. However, the use of Mellin transform methods 15 is extremely cumbersome when logarithms appear in the denominator. Since inverse logarithms cannot be avoided in the spectral function, we need to resort to other means.

We carry out the dispersion integral of the $\Delta \rho_{\text {power }}(u)$ term by splitting the integral in Eq. (7) into three intervals: $\left(0, \lambda^{2}\right),\left(\lambda^{2}, Q^{2}\right)$ and $\left(Q^{2}, \infty\right)$.

The integral over the first interval can be naively expanded in powers of $1 / Q^{2}$, since there are no convergence problems:

$$
\Delta \tilde{K}_{1}(i Q) \equiv \int_{0}^{\lambda^{2}} d u^{2} \frac{\Delta \rho_{\text {power }}(u)}{u^{2}+Q^{2}} \sim \frac{1}{Q^{2}} \sum_{n=0}^{\infty} \frac{(-1)^{n}}{Q^{2 n}} \int_{0}^{\lambda^{2}} d u^{2} u^{2 n} \Delta \rho_{\text {power }}(u) .
$$

In particular, the leading term in $1 / Q^{2}$ is

$$
\Delta \tilde{K}_{1}(i Q) \sim \frac{1}{Q^{2}} \int_{0}^{\lambda^{2}} d u^{2} \Delta \rho_{\text {power }}(u)+\mathcal{O}\left(1 / Q^{4}\right)
$$

In the second interval, we use the asymptotic form of $\Delta \rho_{\text {power }}$ given in Eq. (10b) and obtain (for instance by repeatedly integrating by parts)

$$
\begin{aligned}
\Delta \tilde{K}_{2}(i Q) & \equiv \int_{\lambda^{2}}^{Q^{2}} d u^{2} \frac{\Delta \rho_{\text {power }}(u)}{u^{2}+Q^{2}} \sim \frac{1}{Q^{2}} \sum_{n, \nu=0}^{\infty} a_{n}^{(\nu)}\left\{\frac{(-1)^{n}}{Q^{2 n}} \frac{\left(\ln Q^{2}\right)^{\alpha}-\left(\ln \lambda^{2}\right)^{\alpha}}{\alpha}\right. \\
& \left.+\sum_{\substack{l=0 \\
l \neq n}}^{\infty} \frac{(-1)^{l}}{Q^{2 l}} \frac{\Gamma\left(\alpha,(n-l) \ln \lambda^{2}\right)-\Gamma\left(\alpha,(n-l) \ln Q^{2}\right)}{(n-l)^{\alpha}}\right\}
\end{aligned}
$$

where $\alpha \equiv 1-\nu-\xi$, and $\Gamma[\alpha, z]$ is the incomplete Gamma function. Notice that when $\alpha \equiv$ $1-\nu-\xi=0$ the correct result for the term with $l=n$ is $\ln \frac{\ln Q^{2}}{\ln \lambda^{2}}$, which also corresponds to 
its limiting value: $\lim _{\alpha \rightarrow 0}\left[\left(\ln Q^{2}\right)^{\alpha}-\left(\ln \lambda^{2}\right)^{\alpha}\right] / \alpha=\ln \frac{\ln Q^{2}}{\ln \lambda^{2}}$. We then substitute in the second incomplete Gamma function that appears in Eq. (13) the following asymptotic expression which is valid for $|z| \rightarrow \infty$ and $-3 \pi / 2<\arg z<3 \pi / 2$ :

$$
\Gamma[\alpha, z] \equiv \int_{z}^{\infty} d x x^{\alpha-1} e^{-x} \sim z^{\alpha-1} e^{-z} \sum_{m=0}^{\infty} \frac{(-1)^{m} \Gamma(1-\alpha+m)}{z^{m} \Gamma(1-\alpha)} .
$$

The resulting expression for $\Delta \tilde{K}_{2}(i Q)$ is

$$
\begin{aligned}
\Delta \tilde{K}_{2}(i Q) & \sim \frac{1}{Q^{2}} \sum_{l, \nu=0}^{\infty} \frac{(-1)^{l}}{Q^{2 l}}\left[\sum_{\substack{n=0 \\
n \neq l}}^{\infty} a_{n}^{(\nu)} \frac{\Gamma\left(\alpha,(n-l) \ln \lambda^{2}\right)}{(n-l)^{\alpha}}-a_{l}^{(\nu)} \frac{\left(\ln \lambda^{2}\right)^{\alpha}}{\alpha}\right] \\
& -\frac{1}{Q^{2}} \sum_{n, \nu=0}^{\infty} \frac{a_{n}^{(\nu)}}{Q^{2 n}}\left[\sum_{\substack{l=0 \\
l \neq n}}^{\infty} \sum_{m=0}^{\infty} \frac{(-1)^{l+m}}{(n-l)^{m+1}\left[\ln Q^{2}\right]^{m+1-\alpha}} \frac{\Gamma(m+1-\alpha)}{\Gamma(1-\alpha)}-\frac{(-1)^{n}\left[\ln Q^{2}\right]^{\alpha}}{\alpha}\right],
\end{aligned}
$$

where again $\left(\ln \lambda^{2}\right)^{\alpha} / \alpha$ and $\left(\ln Q^{2}\right)^{\alpha} / \alpha$ should be understood as $\ln \left(\ln \lambda^{2}\right)$ and $\ln \left(\ln Q^{2}\right)$ when $\alpha \equiv 1-\nu-\xi=0$.

Let us notice that in the first line of Eq. (15) any term with $l$ such that the integral $\int_{\lambda^{2}}^{\infty} d u^{2} u^{2 l} \Delta \rho_{\text {power }}(u)$ is finite can be formally identified as

$$
\sum_{\nu=0}^{\infty}\left[\sum_{\substack{n=0 \\ n \neq l}}^{\infty} a_{n}^{(\nu)} \frac{\Gamma\left(\alpha,(n-l) \ln \lambda^{2}\right)}{(n-l)^{\alpha}}-a_{l}^{(\nu)} \frac{\left(\ln \lambda^{2}\right)^{\alpha}}{\alpha}\right]=\int_{\lambda^{2}}^{\infty} d u^{2} u^{2 l} \Delta \rho_{\text {power }}(u) .
$$

In particular, the leading $1 / Q^{2}$ term in Eq. (15) is

$$
\begin{aligned}
\Delta \tilde{K}_{2}(i Q) & \sim \frac{1}{Q^{2}} \sum_{\nu=0}^{\infty}\left[\sum_{n=1}^{\infty} a_{n}^{(\nu)} \frac{\Gamma\left(\alpha, n \ln \lambda^{2}\right)}{n^{\alpha}}-a_{0}^{(\nu)} \frac{\left(\ln \lambda^{2}\right)^{\alpha}}{\alpha}\right] \\
& +\frac{1}{Q^{2}} \sum_{\nu=0}^{\infty} a_{0}^{(\nu)}\left[\sum_{l=1}^{\infty} \sum_{m=0}^{\infty} \frac{(-1)^{l}}{l^{m+1}\left[\ln Q^{2}\right]^{m+1-\alpha}} \frac{\Gamma(m+1-\alpha)}{\Gamma(1-\alpha)}+\frac{\left[\ln Q^{2}\right]^{\alpha}}{\alpha}\right]+\mathcal{O}\left(\frac{1}{Q^{4}}\right) .
\end{aligned}
$$

We can similarly expand the integral over the third interval

$$
\begin{aligned}
\Delta \tilde{K}_{3}(i Q) & \equiv \int_{Q^{2}}^{\infty} d u^{2} \frac{\Delta \rho_{\text {power }}(u)}{u^{2}+Q^{2}} \sim-\frac{1}{Q^{2}} \sum_{n, \nu=0}^{\infty} a_{n}^{(\nu)} \sum_{l=1}^{\infty}\left(-Q^{2}\right)^{l} \frac{\Gamma\left(\alpha,(n+l) \ln Q^{2}\right)}{(n+l)^{\alpha}} \\
& \sim-\frac{1}{Q^{2}} \sum_{n, \nu=0}^{\infty} \frac{a_{n}^{(\nu)}}{Q^{2 n}} \sum_{l=1}^{\infty} \sum_{m=0}^{\infty} \frac{(-1)^{l+m}}{(n+l)^{m+1}\left[\ln Q^{2}\right]^{m+1-\alpha}} \frac{\Gamma(m+1-\alpha)}{\Gamma(1-\alpha)} \\
& \sim-\frac{1}{Q^{2}} \sum_{\nu=0}^{\infty} a_{0}^{(\nu)} \sum_{l=1}^{\infty} \sum_{m=0}^{\infty} \frac{(-1)^{l+m}}{l^{m+1}\left[\ln Q^{2}\right]^{m+1-\alpha}} \frac{\Gamma(m+1-\alpha)}{\Gamma(1-\alpha)}+\mathcal{O}\left(\frac{1}{Q^{4}}\right) .
\end{aligned}
$$

In the end we add the leading $1 / Q^{2}$ contributions from Eqs. (12), (18) and (18) to the corresponding contribution from the naive expansion of $\Delta \rho_{\exp }$ and obtain

$$
\begin{aligned}
\Delta \tilde{K}(i Q) \equiv \int_{0}^{\infty} & d u^{2} \frac{\Delta \rho(u)}{u^{2}+Q^{2}} \sim \frac{\overline{\Delta \rho}}{Q^{2}}+\frac{\left[\ln Q^{2}\right]^{1-\xi}}{Q^{2}} \sum_{\nu=0}^{\infty} \frac{a_{0}^{(\nu)}}{\left[\ln Q^{2}\right]^{\nu}}\left[\frac{1}{1-\xi-\nu}\right. \\
& \left.+2 \sum_{\substack{l=1 \\
m=0}}^{\infty} \frac{(-1)^{l}}{\left[l \ln Q^{2}\right]^{2 m+2}} \frac{\Gamma(2 m+1+\nu+\xi)}{\Gamma(\nu+\xi)}\right]+\mathcal{O}\left(\frac{1}{Q^{4}}\right),
\end{aligned}
$$


where $\overline{\Delta \rho}$ is defined by

$$
\begin{aligned}
\overline{\Delta \rho} & \equiv \int_{0}^{\lambda^{2}} d u^{2} \Delta \rho_{\text {power }}(u)+\int_{0}^{\infty} d u^{2} \Delta \rho_{\exp }(u) \\
& +\sum_{\substack{n=1 \\
\nu=0}}^{\infty} a_{n}^{(\nu)} \frac{\Gamma\left(1-\nu-\xi, n \ln \lambda^{2}\right)}{n^{1-\nu-\xi}}-\sum_{\nu=0}^{\infty} a_{0}^{(\nu)} \frac{\left(\ln \lambda^{2}\right)^{1-\nu-\xi}}{1-\nu-\xi} .
\end{aligned}
$$

Since the integral $\int^{\infty} d x x^{-1}(\ln x)^{-\xi}$ can be finite only if $\xi>1$, we can use Eq. (16) to identify $\overline{\Delta \rho}$ as the zeroth moment of the subtracted spectral function, only when the asymptotic expansion of the subtracted SF in Eq. (10b) yields $\xi>1$. Otherwise, if $\xi \leq 1$, the zeroth moment is infinite, though $\overline{\Delta \rho}$ as defined by Eq. (20) still exists.

\section{E. Exact Sum Rules}

We derive the sum rules by comparing the coefficient of $1 / Q^{2}$ in Eq. (19)

$$
Q^{2} \int_{0}^{\infty} d u^{2} \frac{\Delta \rho(u)}{u^{2}+Q^{2}} \sim \overline{\Delta \rho}+a_{0}^{(0)} \times \begin{cases}\frac{\left[\ln Q^{2}\right]^{1-\xi}}{(1-\xi)}+\mathcal{O}\left(\frac{1}{Q^{2}}, \frac{1}{\left[\ln Q^{2}\right]^{\xi}}\right) & \text { if } \xi \neq 1 \\ \ln \left(\ln Q^{2}\right) & \text { if } \xi=1\end{cases}
$$

and the corresponding coefficient in Eqs. (9)

$$
Q^{2} \Delta \tilde{K}(i Q) \sim c_{1}^{(0)}(\kappa) \Delta\left\langle\left[O_{1}\right]_{\kappa}\right\rangle\left[g^{2}(Q)\right]^{\eta}+\mathcal{O}\left(\frac{1}{Q^{2}},\left[g^{2}(Q)\right]^{\eta+1}\right),
$$

where we are only considering cases with $n=1$ and $d_{1}=2$. Furthermore, we are presently interested in exact sum rules that can be derived with one-loop calculations and, therefore, we only compare leading orders in $1 / \ln Q^{2}$. Then there exist three possibilities, depending on the value of $\eta$, which can be calculated using the OPE.

(1) If $\eta=0$, then $\xi=2$ (or a bigger integer) and

$$
\overline{\Delta \rho}=\int_{0}^{\infty} d u^{2} \Delta \rho(u)=c_{1}^{(0)}(\kappa) \Delta\left\langle\left[O_{1}\right]_{\kappa}\right\rangle .
$$

This is the result one would get by naively expanding $Q^{2} /\left(u^{2}+Q^{2}\right)$ without worrying about the convergence of the moments and disregarding logarithmic corrections in the OPE.

(2) If $\eta>0$, then $\xi=1+\eta$ and

$$
\overline{\Delta \rho}=\int_{0}^{\infty} d u^{2} \Delta \rho(u)=0
$$

(3) If $\eta<0$ (positive powers of $\ln Q^{2}$ in Eq. (8)) or if the term $\ln \ln Q^{2}$ appears in Eq. (8), then $\xi<1$ or $\xi=1$, respectively. This implies that

$$
\overline{\Delta \rho} \neq \int_{0}^{\infty} d u^{2} \Delta \rho(u)=\infty .
$$


We remark that, even in this case when the moment is not finite, the asymptotic expansion is still well defined. It is nice to see that whether the zeroth moment of the subtracted SF exists is reflected directly through the leading power of $g^{2}(Q)$ in the OPE series.

Our main results, Eq. (23) and Eq. (24), can be expressed in physical terms as follows. The zeroth moment of a SF for a current $J$ whose OPE series yields $\eta>0$ is independent of $T$ and $\mu$, while the same moment for a current with $\eta=0$ changes with $T$ and $\mu$ proportionally to the corresponding change(s) of the condensate(s) of the leading operator(s). Although $c_{1}^{(0)}(\kappa)$ and $\Delta\left\langle\left[Q_{1}\right]_{\kappa}\right\rangle$ can separately depend on $\kappa$, their product must be independent of $\kappa$, since the zeroth moment is independent of $\kappa$.

\section{F. Discussion}

At this point several general comments are appropriate:

(1) Our derivation relies on the fact that an asymptotically free theory allows a perturbative expansion at short distances, making practical the use of the OPE and of the RGE. We understand why only short distance physics is involved if we realize that the integral over frequencies reduces Eq. (1) to the ensemble average of the equal-time commutator of the currents. Therefore, results such as Eq. (23) and Eq. (24) are completely determined by the one-loop structure of the theory and the particular current under exam.

(2) Flavor, or other non-dynamical quantum numbers, does not change the expansions at the one-loop level in an essential way. Therefore, one can derive analogous sum rules by using other kind of subtractions, instead of the one we adopted. One such example is given by the exact Weinberg sum rules $(T=0)$ in the chiral limit [16].

(3) The derivation of sum rules for higher moments of the SF requires the complete cancelation of all the lower dimensional operator terms, not just the leading $g^{2}(Q)$. In particular, one also needs current quark mass corrections to the Wilson coefficients. Without appropriate subtractions, higher moments do not even converge [17].

(4) It is essential to properly take into account the logarithmic corrections when deriving exact sum rules, since the logarithmic corrections not only dictate whether $\overline{\Delta \rho}$ satisfy Eq. (23) or Eq. (24) but they also control the very existence of $\overline{\Delta \rho}$ [17. This procedure is in sharp contrast with the usual QCD sum rule approach, where the convergence issue is by-passed by applying the Borel improvement by explicitly introducing a cut-off parameter (the Borel mass).

(5) We believe that the $(T, \mu)$-dependent part of the leading condensate appearing in Eq. (23) does not suffer from the infrared renormalon ambiguity. In fact, only the perturbative term $\tilde{K}_{0}$ can generate contributions to the leading condensate that are dependent on the prescription used to regularize these renormalons. But $\tilde{K}_{0}$ is independent of $T$ and $\mu$ : any prescription dependence cancels out when we make the subtraction in Eq. (7). On the contrary, unless we generalize Eq. (7) and make other subtractions, sum rules that involve non-leading condensates are, in principle, ambiguous.

(6) It is well-known that conserved operators are not renormalized and, barring anomalous violations, verify the same "classical" identities that can be derived at the tree level. This fact is also verified in the sum rules. In fact when both the currents and the operators are conserved $(\eta=0)$ and we obtain the result of Eq. (23), i.e. one can use the "naive 
asymptotic expansion" to derive the sum rule. Conservation of the current alone is not enough to warrant a "classical" identity.

\section{GROSS-NEVEU MODEL}

In the preceding section we have derived sum rules valid for any asymptotically free theory. In this section we illustrate the procedure in the $1+1$ dimensional Gross-Neveu model [18] in the infinite- $N$ limit. On one hand, we can derive the sum rules in Eqs. (23) and (24) by explicitly calculating Wilson coefficients, $\beta$ - and $\Gamma$-functions in the vector and pseudoscalar channels, following the general procedure discussed in section II. On the other hand, since this model is soluble, we can obtain the exact spectral function at arbitrary $(T, \mu)$, and then explicitly verify both that the sum rules are satisfied and that the asymptotic expansion of the spectral function has the form given in Eq. (10b). Moreover, we can also calculate the condensates, and therefore explicitly check that the OPE really matches the asymptotic expansion of the exact spectral integral.

The Lagrangian of the Gross-Neveu model is

$$
\mathcal{L}=\bar{\psi} i \gamma \cdot \partial \psi+\frac{g^{2}}{2 N}\left[(\bar{\psi} \psi)^{2}+\left(\bar{\psi} i \gamma_{5} \psi\right)^{2}\right]
$$

where $\psi$ is a two-component Dirac spinor and has $N$-component in the internal space. Equivalently, we can write

$$
\mathcal{L}=\bar{\psi}\left(i \gamma \cdot \partial-\sigma-i \gamma_{5} \pi\right) \psi-\frac{N}{2 g^{2}}\left(\sigma^{2}+\pi^{2}\right)
$$

where $\sigma$ and $\pi$ are auxiliary fields. The coupling constant $g^{2}$ is independent of $N$ and held fixed in the limit $N \rightarrow \infty$. This model is asymptotically free when $D=1+1$, and the chiral symmetry is dynamically broken at $T=0$ and $\mu=0$ to the leading order in $1 / N$ 18]. In the following we give the exact solution at $N \rightarrow \infty$ with finite $(T, \mu)$. Although formulas are explicitly considered in the symmetry breaking phase, they are also valid in the symmetric phase provided that the vanishing limit of certain condensates (such as the dynamical fermion mass) is properly taken.

We wish to remark on the well-known fact that the limit $N \rightarrow \infty$ here should not be interpreted as a starting point for an expansion of the model at finite $N$, but rather as different model in itself, which is in fact the model we decided to use for the purpose of illustration. Moreover, there exist arguments [19,20 suggesting that this model in the limit $N \rightarrow \infty$ is actually more relevant to $3+1$ phenomenology than the model with finite $N$.

\section{A. Exact solution in the large- $N$ limit}

Since the procedure to obtain the exact solution in the large- $N$ limit is rather standard and some of the intermediate steps can be found in the literature, see for instance Refs. 18,21, 19,6, 22, we only give the definitions and final results. 


\section{Gap equation and phase diagram}

The gap equation and the phase diagram can be derived from the first derivative of the effective potential

$$
\frac{\partial V\left(\sigma^{2}\right)}{\partial \sigma^{2}}=\left.\frac{N}{2 \pi}\left\{\frac{\pi}{g^{2}}+T \int d k \sum_{n} \frac{1}{\left(i \omega_{n}+\mu\right)^{2}-\left(k^{2}+\sigma^{2}\right)}\right\}\right|_{\omega_{n}=(2 n+1) \pi T} .
$$

We introduce a momentum cutoff $\Lambda$ and add the counterterm $\frac{N}{4 \pi} \ln \left(4 \Lambda^{2} / \kappa^{2}\right)$, where $\kappa$ is the subtraction point. Then the renormalized effective potential becomes

$$
\begin{aligned}
\frac{\partial V\left(\sigma^{2}\right)}{\partial \sigma^{2}} & =\frac{N}{2 \pi}\left\{\frac{\pi}{g^{2}}+\frac{1}{2} \ln \frac{\sigma^{2}}{\kappa^{2}}+\int_{0}^{\infty} d k \frac{f\left(\sqrt{k^{2}+\sigma^{2}} ; T, \mu\right)}{\sqrt{k^{2}+\sigma^{2}}}\right\} \\
& =\frac{N}{2 \pi}\left\{\frac{1}{2} \ln \frac{\sigma^{2}}{M^{2}}+\int_{0}^{\infty} d k \frac{f\left(\sqrt{k^{2}+\sigma^{2}} ; T, \mu\right)}{\sqrt{k^{2}+\sigma^{2}}}\right\}
\end{aligned}
$$

where we have defined

$$
f(x ; T, \mu) \equiv \frac{1}{e^{(x+\mu) / T}+1}+\frac{1}{e^{(x-\mu) / T}+1},
$$

and, in the second equality, we have eliminated the subtraction point by introducing the dynamical fermion mass at $(T, \mu)=0$, i.e. $M=\kappa \exp \left(-\pi / g^{2}\right)$. The effective potential is then obtained by integrating Eq. (29):

$$
V\left(\sigma^{2}\right)=\frac{N}{4 \pi}\left\{\sigma^{2}\left(\ln \frac{\sigma^{2}}{M^{2}}-1\right)+4 T \int_{0}^{\infty} d k \ln \left[\frac{\left(1+e^{-(k+\mu) / T}\right)\left(1+e^{-(k-\mu) / T}\right)}{\left(1+e^{-\left(\sqrt{k^{2}+\sigma^{2}}+\mu\right) / T}\right)\left(1+e^{-\left(\sqrt{k^{2}+\sigma^{2}}-\mu\right) / T}\right)}\right]\right\} .
$$

The gap equation is simply given by

$$
\left.\frac{\partial V\left(\sigma^{2}\right)}{\partial \sigma}\right|_{\sigma=m(T, \mu)}=0
$$

The system possesses "metastable states" at those values of $(T, \mu)$ for which the gap equation has more than one minimum solutions: the solution with lowest $V\left(m^{2}(T, \mu)\right)$ selects the true ground state. When $(T, \mu)$ 's are such that $m(T, \mu)=0$ is the only minimum solution to the gap equation, these points in the $(T, \mu)$-plane defines a second order phase transition line, which separate the symmetry-broken phase, i.e. $m(T, \mu) \neq 0$, from the symmetric phase, i.e. $m(T, \mu)=0$. Taking the limit $\sigma=0$ in Eq. (29), after some suitable rewriting, this critical line obeys the following equation

$$
\ln (2)=\frac{1}{2} \int_{0}^{M} \frac{d k}{k} \frac{\sinh (k / T)}{\cosh [(k+\mu) / 2 T] \cosh [(k-\mu) / 2 T]}-\int_{M}^{\infty} \frac{d k}{k} f(k ; T, \mu),
$$

whose numerical solutions are plotted in Fig. 11 in full line. The full line turns into a dotted line at the heavy dot, where another minimum (dash line) appears and becomes lower than 
the minimum given by Eq. (33). Since the mass gap is finite on the dash line (given by the solution of $V\left(\sigma^{2}\right)=V^{\prime}\left(\sigma^{2}\right)=0$ with $\sigma \neq 0$, where prime denote a derivative with respect to $\sigma^{2}$ ), the dash line is a first order phase boundary. The critical temperature at $\mu=0$ is given by $T_{c}=\left(e^{\gamma_{E}} / \pi\right) M \approx 0.566933 M$. The "tricritical point" (the heavy dot in Fig. (1) can be found by imposing that $V^{\prime}\left(\sigma^{2} \rightarrow 0\right)=V^{\prime \prime}\left(\sigma^{2} \rightarrow 0\right)=0: T_{3} \approx 0.318328 M, \mu_{3} \approx$ $0.608221 M$ [19]. When $T=0$ the chemical potential at which the first order phase transition takes place is $\mu_{c}=M / \sqrt{2}$ 《19].

\section{Bubble graph and correlator in space-like region}

The basic ingredient to build up the correlator is the fermion bubble graph. In the Euclidean region the pseudoscalar bubble, i.e. the free bubble graph between two $J_{5} \equiv \bar{\psi} i \gamma_{5} \psi$ currents, can be conveniently expressed as

$$
\Pi(\omega)=\Pi^{(0)}(\omega)+\Delta \Pi(\omega)
$$

where

$$
\left.\Pi^{(0)}(\omega)\right|_{\omega=i Q}=-\frac{N}{\pi} \ln \left(\frac{\kappa}{m}\right)+\frac{N}{2 \pi} \frac{1}{\sqrt{1+4 m^{2} / Q^{2}}} \ln \left(\frac{\sqrt{Q^{2}+4 m^{2}}+\sqrt{Q^{2}}}{\sqrt{Q^{2}+4 m^{2}}-\sqrt{Q^{2}}}\right)
$$

and

$$
\left.\Delta \Pi(\omega)\right|_{\omega=i Q}=\frac{N}{\pi} \int_{0}^{\infty} d k f\left(E_{k} ; T, \mu\right) \frac{E_{k}}{E_{k}^{2}+Q^{2} / 4} .
$$

Here and in the following $\kappa$ is always the renormalization subtraction point, $m=m(T, \mu)$ is the dynamically generated fermion mass, and $E_{k}=\sqrt{m^{2}+k^{2}}$. We shall also use the short-hand notation

$$
\left\langle\langle A\rangle \equiv \frac{1}{2 \pi} \int_{-\infty}^{\infty} \frac{d k}{E_{k}} A(k) f\left(E_{k} ; T, \mu\right) .\right.
$$

For instance, the use of this notation makes possible to write the gap equation as

$$
\ln \frac{\kappa}{m}-\frac{\pi}{g^{2}}=\int_{0}^{\infty} \frac{d k}{E_{k}} f\left(E_{k} ; T, \mu\right)=\pi\left\langle\left\langle E_{k}^{0}\right\rangle\right\rangle .
$$

In the large- $N$ limit, the correlator in pseudoscalar channel is given by the geometric sum of the bubble graph:

$$
\left\langle J_{5} J_{5}\right\rangle_{T, \mu}(\omega)=\Pi(\omega)\left(1+\frac{g^{2}}{N} \Pi(\omega)\right)^{-1} .
$$

In the Euclidean region, we can then expand this correlator in the $Q^{2} \rightarrow \infty$ limit as

$$
\begin{aligned}
\frac{1}{N}\left\langle J_{5} J_{5}\right\rangle_{T, \mu}(i Q) & =\frac{\ln \left(Q^{2} / \kappa^{2}\right) / 2 \pi}{1+g^{2} \ln \left(Q^{2} / \kappa^{2}\right) / 2 \pi}-2 g^{2}(Q) \frac{m^{2} / g^{4}}{Q^{2}}\left(1-\frac{g^{2}(Q)}{2 \pi}\right) \\
& +\left(\frac{g^{2}(Q)}{g^{2}}\right)^{2} \frac{4}{Q^{2}}\left(\left\langle\left\langle E_{k}^{2}\right\rangle\right\rangle-\frac{m^{2}}{2}\left\langle\left\langle E_{k}^{0}\right\rangle\right\rangle\right)+\mathcal{O}\left(\frac{1}{Q^{4}}\right)
\end{aligned}
$$


where we have introduced the running coupling constant

$$
g^{2}(Q) \equiv \frac{g^{2}}{1+\left(g^{2} / 2 \pi\right) \ln \left(Q^{2} / \kappa^{2}\right)},
$$

and used the gap equation Eq. (36).

We can recognize the first term in Eq. (38) as the perturbative contribution at $(T, \mu)=0$, $\left\langle J_{5} J_{5}\right\rangle^{(0)}(i Q)$, which corresponds to the term $\tilde{K}_{0}(i Q)$ in the generic OPE expansion shown in section [1]. The other two terms in Eq. (38) can be interpreted as contributions from the condensates. As we will verify later, in fact, $m^{2} / g^{4}=\left\langle\left[(\bar{\psi} \psi)^{2}\right]\right\rangle_{T, \mu}$, while $\left\langle\left\langle E_{k}^{2}\right\rangle\right\rangle-$ $\frac{1}{2} m^{2}\left\langle\left\langle E_{k}^{0}\right\rangle\right\rangle=\left\langle\left[\theta_{00}\right]\right\rangle_{T, \mu}$ is the condensate of the energy-momentum tensor. We shall see that the perturbative contribution and the coefficients of the condensates are indeed the ones obtained by the OPE of $J_{5} J_{5}$, and therefore the expansion of the correlator shown in Eq. (38) is of the form of the OPE.

Note that in defining the condensates we have absorbed factors of $N$ in order to simplify the notation.

\section{Spectral function}

To obtain the spectral function we need the bubble graph in the time-like region $\omega^{2}>$ $4 m^{2}$ :

$$
\frac{1}{N} \operatorname{Im}(\Pi(\omega))=\epsilon(\omega) \frac{\theta\left(\omega^{2}-4 m^{2}\right)}{4 \sqrt{1-4 m^{2} / \omega^{2}}} \times\left\{\tanh \left(\frac{|\omega| / 2+\mu}{2 T}\right)+\tanh \left(\frac{|\omega| / 2-\mu}{2 T}\right)\right\}
$$

and

$$
\frac{1}{N} \operatorname{Re}(\Pi(\omega))=-\frac{1}{g^{2}}+\frac{\tanh ^{-1} \sqrt{1-4 m^{2} / \omega^{2}}}{\pi \sqrt{1-4 m^{2} / \omega^{2}}}+\frac{\omega^{2}}{4 \pi} \mathcal{P} \int_{0}^{\infty} \frac{d k}{E_{k}} \frac{f\left(E_{k} ; T, \mu\right)}{E_{k}^{2}-\omega^{2} / 4}
$$

where $\mathcal{P} \int$ stands for the principle value of the integral.

The complete spectral function has the form

$$
\rho(\omega)=\pi \delta\left(\omega^{2}-m_{\pi}^{2}\right) \frac{N g_{\pi}^{2}(T)}{g^{4}}+\theta\left(\omega^{2}-4 m^{2}\right) \rho_{\text {cont }}(\omega) .
$$

The pole contribution comes from the bound state (the pion), whose mass is the solution to the equation

$$
\frac{N}{g^{2}}+\left.\Pi(\omega)\right|_{\omega=m_{\pi}}=0
$$

in the region $(0,2 m)$. This equation has in fact solution $m_{\pi}=0$, and the coupling constant of this massless pion to its constituents is given by

$$
g_{\pi}^{2}(T)=\left(\frac{\partial}{\partial \omega^{2}} \Pi(\omega) / N\right)_{\omega^{2}=m_{\pi}^{2}=0}^{-1} .
$$


The continuum part of the spectral function is related to the bubble $\Pi(\omega)$ in the time-like region through

$$
\rho_{\text {cont }}(\omega)=\frac{\operatorname{Im} \Pi(\omega)}{\left(1+g^{2} \operatorname{Re} \Pi(\omega) / N\right)^{2}+\left(g^{2} \operatorname{Im} \Pi(\omega) / N\right)^{2}} .
$$

In order to get an idea of the dependence of the spectral function on $(T, \mu)$, we plot $\rho(\omega)$ for several typical values of $(T, \mu)$, which are indicated in the phase diagram in Fig. 1. In Fig. 2 we show $\rho(\omega)$ for values of $(T, \mu)$ that go from the symmetry-broken phase to the symmetric phase through the second order line, while in Fig. 3 we show $\rho(\omega)$ as $(T, \mu)$ go through the first order boundary. We immediately notice that the behavior of the spectral function changes drastically near the second order phase boundary. This is a clear reflection of the low energy critical phenomena related to the continuous phase transition. In fact, the pseudoscalar correlator (in the symmetric phase only, since it is then degenerate with the scalar correlator) at $Q^{2}=0$ can be regarded as the chiral susceptibility, and should diverge as $\left|T-T_{c}\right|^{-\gamma}$ (with $\gamma=1$ in this model). At the boundary of the first order phase transition (Fig. 3), but still relatively close to the "tricritical point", the same qualitative deformation is present, since a weak first order transition shares certain qualitative features of a second order transition, even if we do not expect any real divergence.

It is this kind of dramatic behavior of the spectral function near phase transitions that makes practical parameterizations nearly impossible. The chiral restoration in QCD is expected to be either continuous, or at least a smooth crossover, and the spectral functions in scalar and pseudoscalar channels should show a behavior qualitatively similar to the Gross-Neveu model, i.e. strong peak right above the origin near the phase transition. We

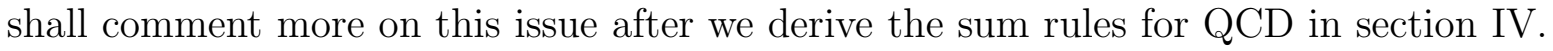

\section{Recovering the OPE series}

It is instructive to expand $\rho(\omega)$ in the limit $\omega^{2} \rightarrow \infty$. We shall see that this asymptotic expansion is of the form assumed in section [1]. Furthermore, once substituted in the dispersion integral, it generates the OPE series of Eq. (38) according to the procedure developed in section [II.

To the leading order in $1 / \omega^{2}$, we find

$$
\frac{g^{2}}{N} \operatorname{Im}(\Pi(\omega)) \sim g^{2} \epsilon(\omega)\left\{\frac{1}{2}+\frac{m^{2}}{\omega^{2}}+\mathcal{O}\left(\frac{1}{\omega^{4}}, e^{-|\omega| / 2 T}\right)\right\}
$$

and

$$
1+\frac{g^{2}}{N} \operatorname{Re}(\Pi(\omega)) \sim \frac{g^{2}}{g^{2}(\omega)}\left\{1+\frac{1}{\omega^{2}}\left[2 m^{2}\left(1-\frac{g^{2}(\omega)}{2 \pi}\right)-4 g^{2}(\omega)\left\langle\left[\theta_{00}\right]\right\rangle_{T, \mu}\right]+\mathcal{O}\left(\frac{1}{\omega^{4}}\right)\right\},
$$

which leads to the following asymptotic form for the continuum part of the spectral function

$$
\begin{aligned}
\frac{1}{N} \rho_{\text {power }}(\omega) & \sim \frac{1}{2} \frac{\left(g^{2}(\omega) / g^{2}\right)^{2}}{1+g^{4}(\omega) / 4}-\frac{g^{4}(\omega)}{\omega^{2}}\left\langle\left[(\bar{\psi} \psi)^{2}\right]\right\rangle_{T, \mu} \\
& +\frac{4 g^{2}(\omega)}{\omega^{2}}\left(\frac{g^{2}(\omega)}{g^{2}}\right)^{2}\left\langle\left[\theta_{00}\right]\right\rangle_{T, \mu}\left\{1+\mathcal{O}\left(g^{2}(Q)\right)\right\}
\end{aligned}
$$


Here we only concern with the $g^{2}(Q)$-dependent terms and postpone the pure $1 / Q^{2}$ terms (related to $\Delta \rho_{\exp }$ ) to a later subsection. We recognize again the first term in Eq. (48 as the asymptotic perturbative spectral function. Moreover, the $(T, \mu)$-dependent part of Eq. (48) has indeed the general form of Eq. (10b).

In connection with the comments we made after Eq. (10b), we point out that Eq. (48) has been obtained by expanding the spectral function in the limit $Q^{2} \rightarrow \infty$ at fixed $T$. Had we made instead a high- $T$ expansion $(T \rightarrow \infty)$ at fixed $Q^{2}$, we would have obtained, for instance, $\ln \left(T^{2} / \omega^{2}\right)$ from the third term of Eq. (41).

Upon substituting Eq. (48) in the dispersion integral, and using the identity

$$
\int \frac{d \omega^{2}}{\omega^{2}+Q^{2}} \frac{g^{2 \nu}(\omega)}{\omega^{2}} \sim \frac{2 \pi}{1-\nu} \frac{g^{2(\nu-1)}(Q)}{Q^{2}}\left\{1+\mathcal{O}\left(g^{2}(Q)\right)\right\}
$$

where $\nu>1$ and we have kept only the $g^{2}(Q)$-dependent part, we find the following series to the leading order in $g^{2}(Q)$,

$$
\int \frac{d \omega^{2}}{\omega^{2}+Q^{2}} \frac{\rho(\omega)}{N} \sim \frac{\left\langle J_{5} J_{5}\right\rangle^{(0)}(Q)}{N}-2 g^{2}(Q) \frac{\left\langle\left[(\bar{\psi} \psi)^{2}\right]\right\rangle_{T, \mu}}{Q^{2}}+4\left(\frac{g^{2}(Q)}{g^{2}}\right)^{2} \frac{\left\langle\left[\theta_{00}\right]\right\rangle_{T, \mu}}{Q^{2}}+\cdots
$$

This result is in agreement with the OPE series of Eq. (38), which has been directly expanded from the correlator in the Euclidean region.

The fact that no pure $1 / Q^{2}$ term appears in Eq. (49) implies that the zeroth moment of the subtracted spectral function vanishes: $\overline{\Delta \rho}=0$. The zeroth moment of the spectral function without the subtraction is not convergent, because of the contribution from the perturbative term, even if one can define it through a proper analytic continuation that yields $\bar{\rho}=0$.

\section{B. Direct solution in terms of the OPE}

We have just verified that in the Gross-Neveu model the exact correlation function can indeed be expanded in an asymptotic series precisely in the form of the OPE. Now we shall calculate the Wilson coefficients, $\beta$-function and appropriate anomalous dimensions for the OPE, and verify that they match the coefficients and exponents of this asymptotic series. Furthermore, though it is beyond the scope of the OPE and RGE, we shall also calculate the condensates. In this way, we explicitly verify that the asymptotic expansion of the exact correlation function is identical to the OPE.

\section{One-loop calculation}

The one-loop $\beta$-function of the $1+1$ dimensional Gross-Neveu model has already been calculated in the original paper [18]: $\beta=-g^{4} / 2 \pi$, i.e. according to our notation $b=1 / 2 \pi$.

The anomalous dimensions for pseudoscalar current $J_{5} \equiv \bar{\psi} i \gamma_{5} \psi$ and four-quark operator $O_{4}=(\bar{\psi} \psi)^{2}$ can be calculated using the Feynman diagrams shown in Fig. Ha and Fig. đbb, respectively. According to the notation of Eq. (5) we find $\gamma_{J_{5}}=1 / \pi$, and $\gamma_{O_{4}}=2 / \pi$. The energy-momentum conservation makes the anomalous dimension for $\theta_{00}$ vanish, i.e. $\gamma_{\theta_{00}}=0$. 
The relevant Wilson coefficients corresponding to operators $O_{4}$ and $\theta_{00}$ can be calculated, to leading order in $g^{2}(Q)$, using the Feynman diagrams shown in Fig. 5a and Fig. 5b $\mathrm{b}$, respectively. The resulting coefficient are $c_{O_{4}}=-2 g^{2}(Q)\left(\nu_{O_{4}}^{(0)}=1\right.$ according to notation in Eq. (9)) and $c_{\theta_{00}}=4\left(\nu_{\theta_{00}}^{(0)}=0\right)$.

These results lead to the exponents $\eta_{O_{4}}=1+\left(2 \gamma_{J_{5}}-\gamma_{O_{4}}\right) / 2 b=1$ and $\eta_{\theta_{00}}=\left(2 \gamma_{J_{5}}-\right.$ $\left.\gamma_{\theta_{00}}\right) / 2 b=2$, which are precisely what we found in Eq. (49).

These explicit one-loop calculations exactly match the solution in Eq. (49).

At last a comment on the one-loop calculation: this result is exact to the leading order in $1 / N$ and only involves insertion of the quark-bubble chain. Since the quark-bubble behaves like $\propto \ln Q^{2}$, this insertion is equivalent to substituting the coupling constant $g^{2}$ with the running coupling constant $g^{2}(Q)$. So one can easily identify those sets of Feynman graphs whose sum leads to the solution of the RGE in Eq. (4).

\section{Condensates}

The OPE itself does not specify how the relevant condensates are calculated, since the OPE is only a RGE-improved perturbative procedure. The calculation of the condensates can be done only in a non-perturbative context. Here, we carry out this calculation using the $1 / N$ expansion.

The bare quark condensate $\langle\bar{\psi} \psi\rangle_{T, \mu}$ is given by the dynamical quark tadpole graph

$$
\langle\bar{\psi} \psi\rangle_{T, \mu}=(-i T) \int^{\Lambda} \frac{d k}{2 \pi} \sum_{n} \operatorname{Tr} \frac{i}{k \cdot \gamma-m}=2 m T \int^{\Lambda} \frac{d k}{2 \pi} \sum_{n} \frac{1}{\left(i \omega_{n}+\mu\right)^{2}-\left(k^{2}+m^{2}\right)} .
$$

Using the standard contour integral technique to carry out the Matsubara frequency sum, we obtain

$$
\langle\bar{\psi} \psi\rangle_{T, \mu}=m\left\langle\left\langle E_{k}^{0}\right\rangle\right\rangle-\frac{m}{\pi} \ln \left(\frac{2 \Lambda}{m}\right) .
$$

The renormalized quark condensate is obtained by replacing $2 \Lambda$ with the subtraction point $\kappa$ in the above equation

$$
\langle[\bar{\psi} \psi]\rangle_{T, \mu}=m\left\langle\left\langle E_{k}^{0}\right\rangle\right\rangle-\frac{m}{\pi} \ln \left(\frac{\kappa}{m}\right)=-\frac{m}{g^{2}} .
$$

In the last step we have used the gap equation Eq. (36). Notice that the renormalization of this condensate is not independent of the one carried out for the effective potential. In fact, the $\sigma$-field is just an auxiliary field: $\sigma=-g^{2} \bar{\psi} \psi$. Therefore, the gap equation gives us not only the expectation value of the $\sigma$-field, but also of $\bar{\psi} \psi:\langle\sigma\rangle_{T, \mu} \equiv m(T, \mu)=-g^{2}\langle[\bar{\psi} \psi]\rangle_{T, \mu}$. So the renormalizations of $\bar{\psi} \psi$ and $g^{2}$ are related.

The four-quark condensate factorizes in the large- $N$ limit, and we find $\left\langle\left[(\bar{\psi} \psi)^{2}\right]\right\rangle_{T, \mu}=$ $\langle[\bar{\psi} \psi]\rangle_{T, \mu}^{2}=m^{2} / g^{4}$.

The bare kinetic energy expectation value is given by

$$
\left\langle\bar{\psi} i \gamma_{0} \partial_{0} \psi\right\rangle_{T, \mu}=(-i T) \int^{\Lambda} \frac{d k}{2 \pi} \sum_{n} \operatorname{Tr} \gamma_{0} k_{0} \frac{i}{k \cdot \gamma-m}=2 T \int^{\Lambda} \frac{d k}{2 \pi} \sum_{n} \frac{\left(i \omega_{n}+\mu\right)^{2}}{\left(i \omega_{n}+\mu\right)^{2}-\left(k^{2}+m^{2}\right)}
$$


After removing the $(T, \mu)$-independent volume and quadratic divergences, and introduced a subtraction point for the logarithmic divergence the renormalized kinetic energy becomes

$$
\left\langle\left[\bar{\psi} i \gamma_{0} \partial_{0} \psi\right]\right\rangle_{T, \mu}=\left\langle\left\langle E_{k}^{2}\right\rangle\right\rangle-\frac{m^{2}}{2 \pi} \ln \left(\frac{\kappa}{m}\right) .
$$

After subtracting the trace term from the kinetic energy, we finally obtain the expectation value of the traceless energy-momentum tensor

$$
\left\langle\left[\theta_{00}\right]\right\rangle_{T, \mu} \equiv\left\langle\left[\bar{\psi} i \gamma_{0} \partial_{0} \psi-\frac{1}{2} m \bar{\psi} \psi\right]\right\rangle_{T, \mu}=\left\langle\left\langle E_{k}^{2}\right\rangle\right\rangle-\frac{m^{2}}{2}\left\langle\left\langle E_{k}^{0}\right\rangle\right\rangle
$$

In the symmetric phase $(m=0)$, the expectation value of the energy-momentum tensor has the very simple form at $\mu=0$

$$
\left\langle\left[\theta_{00}\right]\right\rangle_{T, \mu=0}=\frac{\pi}{6} T^{2}
$$

One can verify that inserting these condensates in the OPE, whose coefficients and exponents we have already calculated, reproduces the asymptotic expansion of the exact correlator in Eq. (38).

\section{Explicit verification of the exact sum rule}

Let us first notice that the sum rule $\int d \omega^{2} \Delta \rho(\omega)=0$ converges very slowly. In fact, the subtracted spectral function has the leading behavior

$$
\Delta \rho(\omega) \equiv \Delta \rho_{2}(\omega)+\mathcal{O}\left(\frac{1}{\omega^{4}}\right)
$$

where

$$
\Delta \rho_{2}(\omega)=\frac{4}{\omega^{2}} \frac{g^{4}(\omega)}{g^{4}}\left\{-\frac{\Delta m^{2}}{g^{4}(\omega)+4}+\frac{4 g^{2}(\omega)}{\pi} \frac{\Delta\left(m^{2}+4 \pi\left\langle\left[\theta_{00}\right]\right\rangle_{T, \mu}\right)}{g^{4}(\omega)+4}\right\} .
$$

Since $g^{2}(\omega)=\pi / \ln (\omega / M)$, the leading behavior in Eq. (58) is $\Delta \rho_{2}(\omega) \sim\left[\omega^{2}(\ln \omega)^{2}\right]^{-1}$, and it is clear that the sum rule only converges logarithmically, i.e. $\lim _{A^{2} \rightarrow \infty} \int_{0}^{A^{2}} d \omega^{2} \Delta \rho(\omega) \sim$ $1 / \ln A^{2}$.

Moreover, it is only thanks to the the logarithmic corrections that the sum rule is finite. Had we disregarded this corrections, we would have found $\Delta \rho_{2}(\omega) \sim \omega^{-2}$, and the zeroth moment of the subtracted spectral function would not exist.

Since the exact spectral function cannot be integrated analytically, we verify the exact sum rule numerically. We take advantage of the fact that the integral of $\Delta \rho_{2}(\omega)$ in Eq. (58) can be done analytically to improve the numerical convergence of the integral. We rewrite the sum rule as

$$
\int_{0}^{A^{2}} d \omega^{2} \Delta \rho(\omega)=-\int_{A^{2}}^{\infty} d \omega^{2} \Delta \rho(\omega)=-\int_{A^{2}}^{\infty} d \omega^{2} \Delta \rho_{2}(\omega)+\mathcal{O}\left(\frac{1}{A^{2}}\right),
$$


where the integral of $\Delta \rho_{2}(\omega)$ can be done analytically and we obtain

$$
\int_{0}^{A^{2}} d \omega^{2} \Delta \rho(\omega)=\frac{\pi}{g^{4}}\left\{2 \Delta m^{2}\left(\frac{2}{\pi} \tan ^{-1} \frac{2 \ln A}{\pi}-1\right)+\frac{\Delta\left(m^{2}+4 \pi\left\langle\left[\theta_{00}\right]\right\rangle_{T, \mu}\right)}{(\ln A)^{2}+\pi^{2} / 4}\right\}+\mathcal{O}\left(\frac{1}{A^{2}}\right) .
$$

This formula can be easily verified numerically with high accuracy, since the error is now $1 / A^{2}$, rather than $1 / \ln A$. It is reminded that $\Delta \rho$ in the above equation should be obtained from the complete SF in Eq. (42), including both $\Delta \rho_{\text {power }}$ and $\Delta \rho_{\text {exp }}$.

\section{Correlator in vector channel and baryon number susceptibility}

There are several reasons to study the correlator in the vector channel in the GrossNeveu model. First, the vector channel gives us an example where the zeroth moment of the subtracted SF does not exist, but the asymptotic procedure can still be carried out. Moreover, in connection with the fact that the vector current is conserved, this spectral function is not affected by logarithmic corrections, and we can use it to illustrate the use of the Mellin transform method to obtain the asymptotic expansion. In addition, very much like in the QCD case, we shall see that the baryon number susceptibility changes drastically near the chiral restoration transition, even if we do not expect any real singularity associated with critical phenomena in the vector channel. For convenience, we only consider the case of $\mu=0$.

\section{Vector correlator in Euclidean region}

The Feynman graphs that contribute to the vector correlator are depicted in Fig. 6. Note that if there were no mixing between vector and pseudoscalar channel, which in fact is only present in $1+1$ dimensions, the sole contribution would come from the first graph. The total contribution from all the graphs in Fig. 6 is

$$
\left\langle J_{\alpha} J^{\alpha}\right\rangle_{T, \mu}(i Q)=\frac{1}{\pi}\left\{1-\frac{8 m^{2}}{\sqrt{Q^{2}\left(Q^{2}+4 m^{2}\right)}} \tanh ^{-1} \sqrt{\frac{Q^{2}}{Q^{2}+4 m^{2}}}+2 m^{2} \int_{0}^{\infty} \frac{d k}{E_{k}} \frac{f\left(E_{k} ; T, 0\right)}{E_{k}^{2}+Q^{2} / 4}\right\} .
$$

We define the spectral function in vector channel as

$$
\left\langle J_{\alpha} J^{\alpha}\right\rangle_{T, \mu}(i Q)=\frac{1}{\pi}\left\{1-\int_{0}^{\infty} d \omega^{2} \frac{\tilde{\rho}(\omega)}{\omega^{2}+Q^{2}}\right\} .
$$

Then we find the following explicit expression for $\tilde{\rho}$

$$
\tilde{\rho}(\omega)=\frac{4 m^{2} \theta\left(\omega^{2}-4 m^{2}\right)}{\sqrt{\omega^{2}\left(\omega^{2}-4 m^{2}\right)}}\left(1-\frac{2}{e^{|\omega| / 2 T}+1}\right) .
$$

It is clear that leading asymptotic behavior of the spectral function is $\tilde{\rho}(\omega) \sim 1 / \omega^{2}$, which implies that the zeroth moment of the spectral function does not exist (it is divergent). In 
the section 1 II we have shown that, corresponding to this divergence, we must find negative powers of $g^{2}(Q)$ (positive power of $\ln \left(Q^{2}\right)$ ) in the OPE series.

When there are no explicit logarithms in $\tilde{\rho}(\omega)$, it is easier to make the asymptotic expansion by using the Mellin transform method, and in particular the convolution property of the Mellin transform [15],

$$
\int_{0}^{\infty} d t f(t) h(t)=\frac{1}{2 \pi i} \int_{c-i \infty}^{c+i \infty} d z M[h ; z] M[f ; 1-z]
$$

where $M[h ; z]$ is the Mellin transform of $h(t)$, defined by

$$
M[h ; z] \equiv \int_{0}^{\infty} d t t^{z-1} h(t) .
$$

The value of $c$ in Eq.(64) depends on the asymptotic behaviors of $h(t)$ at $t \rightarrow \infty$ and $f(t)$ at $t \rightarrow 0$ respectively; details can be found, for instance, in Chapter 4 of Ref. [15].

Now let us illustrate how to use this method to expand the power part of the spectral function (the part containing exponential dumping factors can be expanded naively)

$$
\tilde{\rho}_{\text {power }}(\omega)=\frac{4 m^{2} \theta\left(\omega^{2}-4 m^{2}\right)}{\sqrt{\omega^{2}\left(\omega^{2}-4 m^{2}\right)}}
$$

whose Mellin transform is

$$
M\left[\tilde{\rho}_{\text {power }} ; z\right]=\left(4 m^{2}\right)^{z} \sqrt{\pi} \Gamma(1-z) / \Gamma(3 / 2-z) .
$$

Using the convolution, Eq. (64), and the Mellin transform of $1 /\left(u^{2}+Q^{2}\right)$

$$
M\left[\left(u^{2}+Q^{2}\right)^{-1} ; 1-z\right]=\pi\left(Q^{2}\right)^{-z} / \sin \pi(1-z),
$$

we find (in this case $c$ in Eq.(64) obeys $0<c<1$ )

$$
\begin{aligned}
\int_{0}^{\infty} d \omega^{2} \frac{\tilde{\rho}_{\text {power }}}{\omega^{2}+Q^{2}} & =-\sum_{n=1}^{\infty} \operatorname{Res}\left[\left(\frac{4 m^{2}}{Q^{2}}\right)^{z} \frac{\sqrt{\pi} \Gamma(1-z)}{\Gamma(3 / 2-z)} \frac{\pi}{\sin \pi(1-z)}\right]_{z=n} \\
& =\frac{4 m^{2}}{Q^{2}} \ln \left(\frac{Q^{2}}{m^{2}}\right)-\frac{8 m^{4}}{Q^{4}}\left[1+\ln \left(\frac{Q^{2}}{m^{2}}\right)\right]+\mathcal{O}\left(\frac{1}{Q^{6}}\right) .
\end{aligned}
$$

Similarly to the pseudoscalar correlator, it is possible to check this result in two ways. We can directly expand the power part (temperature independent part) of Eq. (61), and we can derive the $\operatorname{OPE}\left(\gamma_{V}=0\right.$ and $c_{O_{4}}=8 g^{2}(Q)$ for the $1 / Q^{2}$ term).

As promised, there indeed appears a positive power of $\ln \left(Q^{2}\right)$ in the OPE series. At the same time, the singularities of $M\left[\tilde{\rho}_{\text {power }} ; z\right]$ at $z=$ positive integer hints the fact that all the non-negative integer moments of $\tilde{\rho}_{\text {power }}$ do not exist. On the other hand, the asymptotic expansion in Eq. (69) is well defined. Even though the vector current is conserved, due to the fact that the anomalous dimension of $\mathrm{O}_{4}$ is not zero, the correct result can not be obtained by a naive asymptotic expansion in this case, in accordance with the remark number (6) at the end of section II.

This same result could have been obtained also by the general method developed in section 【1, but it should be clear by now that the use of the Mellin transform, when possible, is more straightforward and require less labour. 


\section{Baryon number susceptibility}

The baryon number susceptibility $\left(\chi_{\mathrm{BN}}\right)$ is defined as the vector correlator in the limit $Q^{2} \rightarrow 0$. From Eq. (61) we find

$$
\chi_{\mathrm{BN}} \equiv \pi\left\langle J_{\alpha} J^{\alpha}\right\rangle_{T}(i Q \rightarrow 0)=-1+2 m^{2} \int_{0}^{\infty} \frac{d k}{E_{k}^{3}} \frac{2}{e^{E_{k} / T}+1} .
$$

Notice that since we have contracted the Lorentz indices when defining our vector correlator, $\chi_{\mathrm{BN}}$ is nonpositive definite. It is also possible that our definition of $\chi_{\mathrm{BN}}$ differs from others by an additive constant, which is irrelevant to the temperature dependence we are considering. In the symmetric phase, the limit $m \rightarrow 0$ should be take with some care: the result is $\chi_{\mathrm{BN}}=1$, when $T>T_{c}$. The complete temperature dependence of $\chi_{\mathrm{BN}}$ is displayed in Fig. $0 \mathrm{~b}$. As expected, there is no divergence or critical phenomena in this channel. Nevertheless, we do see a sudden rising of $\chi_{\mathrm{BN}}$ in correspondence to the relatively rapid drop of the dynamical quark mass, which we show in Fig. 7a for comparison, near the phase transition region.

\section{APPLiCATION TO QCD}

After a lengthy detour to the Gross-Neveu model let us now back to the main interest of our work, to derive exact sum rules and to explore the corresponding phenomenological consequences in QCD. As we will see, QCD shares many of the qualitative features observed in the Gross-Neveu model, implying the simple fact that any drastic change induced by a phase transition or rapid crossover would necessarily be reflected through the spectral function in proper channels at low frequencies, independent of the details of the model.

\section{A. Sum Rules}

Let us consider four correlators of mesonic currents in QCD. The correlation between scalar currents and the one between pseudoscalar currents involve non-conserved operators, $J_{S}=\bar{\psi} \psi$ and $J_{P}=\bar{\psi} \gamma_{5} \psi$, whose anomalous dimensions are $\gamma_{J_{S}}=\gamma_{J_{P}}=1 / 4 \pi^{2}$. One the other end, correlators between vector currents and axial-vector currents involve conserved quantities, $J_{V}=\bar{\psi} \gamma_{\mu} \psi$ and $J_{A}=\bar{\psi} \gamma_{\mu} \gamma_{5} \psi$, whose anomalous dimensions vanish $\left(\gamma_{V}=\gamma_{A}=\right.$ $0)$.

In QCD there exist several dimension-four operators, but all of them have non-positive anomalous dimensions, therefore the two correlators between nonconserved currents have $\eta_{n} \geq\left(2 \gamma_{J}-\gamma_{n}\right) / 2 b>0$ and Eq. (24) applies, i.e. the zeroth moments of their SF's are independent of $T$ and $\mu$.

On the other hand, the two conserved currents have, in correspondence with conserved operators $\eta_{n}=0$ and a generalization of Eq. (23) applies. In this case in fact there are three dimension-four operators with zero anomalous dimension. Two of these operators are

Lorentz scalars: $m \bar{\psi} \psi$ and $\alpha_{s} G^{2} \equiv \alpha_{s} \operatorname{tr}\left(G_{\mu \nu} G^{\mu \nu}\right)$, while the third is the energy-momentum tensor:

$$
\theta_{\mu \nu}=\frac{1}{4} \bar{\psi}\left(\gamma_{\mu} i \stackrel{\leftrightarrow}{D}_{\nu}+\gamma_{\nu} i \stackrel{\leftrightarrow}{D}_{\mu}-m g_{\mu \nu}\right) \psi+2 \operatorname{tr}\left(G_{\mu \alpha} G_{\nu}{ }^{\alpha}\right)-\frac{1}{2} g_{\mu \nu} G^{2}
$$


The sum rules for the vector and axial-vector currents (with the Lorentz indices contracted) are:

$$
\int_{0}^{\infty} d u^{2} \Delta \rho(u)=a \Delta\langle[m \bar{\psi} \psi]\rangle+\frac{\Delta\left\langle\left[\alpha_{s} G^{2}\right]\right\rangle}{2 \pi}+8 \Delta\left\langle\left[\theta_{00}\right]\right\rangle,
$$

with $a=6$ and $a=-10$ for vector and axial-vector cases, respectively.

Obviously, the general remarks at the end of section II apply to the sum rules derived above. However, there is a new feature of QCD which may not be shared by all asymptotically free theories. It is known that QCD OPE series has explicit instanton induced corrections that cannot be related to condensates of some local operators. But these exact sum rules are not explicitly affected by this problem, although the value of the condensates certainly have instanton contributions. The reason is that the instanton singularities in the Borel-plane are located on the positive axis starting at $8 \pi^{2}$, which in turn implies that the explicit contribution of instantons to correlation functions are of higher order than $1 / Q^{2}$ [23] in the OPE series.

\section{B. Phenomenological Consequences}

Finally, let us discuss some of the phenomenological consequences of these exact sum rules. The power of these exact sum rules rests on their generality, in the sense that there is no assumptions on the functional form of the SF's and they can be applied anywhere in the $(T, \mu)$-plane.

First let us consider the pseudoscalar channel. The sum rule $\overline{\Delta \rho}=0$ implies that, in the broken-chiral-symmetry phase, the change of the pion pole induced by $T$ or $\mu$ is exactly compensated by a corresponding change of the continuum part of the SF.

Next let us consider the scalar correlation function at zero frequency, i.e. the chiral susceptibility (a measure of the fluctuation of the chiral order parameter),

$$
\chi(T, \mu) \equiv \int d^{4} x \theta\left(x_{0}\right)\left\langle\left[J_{S}(x), J_{S}(0)\right]\right\rangle_{T, \mu}=\int_{0}^{\infty} d u^{2} \frac{\rho(u ; T, \mu)}{u^{2}},
$$

which diverges when $(T, \mu)$ approaches the phase boundary, provided the chiral restoration is a continuous transition. The divergence of the chiral susceptibility near the phase transition can only be produced in Eq. (73) by singularities very close to the origin; singularities not near the origin are not compatible with the sum rule $\overline{\Delta \rho}=0$. Thus, when approaching the phase boundary in the $(T, \mu)$ plane, the threshold of the spectral function vanishes (since there is no massless pole in the chirally symmetric phase), and a strong peak develops right above threshold. In the chirally symmetric phase the pseudoscalar and scalar channels are degenerate, therefore we expect the same behavior for the pseudoscalar SF. This strong peak in the pseudoscalar and scalar SF's, which is intimately connected with critical phenomena of a diverging susceptibility and correlation length near the phase transition, can be interpreted as some kind of quasi-particle, thus confirming the qualitative picture, originally proposed in the context of the Nambu-Jona-Lasinio model [24], of the appearance of soft modes near the chiral phase transition. 
We wish to remark that the sum rule has been used only to exclude the logical possibility of the SF developing singularities at finite energies. But the sum rule is not a necessary condition for the appearance of a peak. The presence of a second order phase transition (infinite correlation length) is the real physical cause of the peak. Nevertheless, one should not assume that if the phase transition is not second order the zero- $T$ or perturbative spectral function is a good approximation to the real spectral function. Even if the chiral restoration turns out to be a cross-over or weak first order transition (finite but large correlation), as the lattice data seem to indicate [25], we still expect the same qualitative features, though less pronounced: a peak develops but it does not actually diverge at the transition. Our calculation in the Gross-Neveu model is a clear illustration of this situation. While Fig. (2) shows the divergent peak of the spectral function near the second order boundary, we see in Fig. (3) that the spectral function is also strongly peaked near the first order transition as long as we are not too far away from the "tricritical point".

We believe that similar results hold in the vector and axial-vector channels, even if our conclusions cannot be as strong as in the previous case, because of two main differences. The first is that now $\overline{\Delta \rho} \neq 0$. This problem is not very serious, since we only need that $\overline{\Delta \rho}$ is not singular crossing the phase boundary. And this can still be argued by means of the sum rule in Eq. (72) and the fact that, for a continuous phase transition, the changes of the thermal energy $\Delta\left\langle\left[\theta_{00}\right]\right\rangle$, and of both condensates $\Delta\langle[m \bar{\psi} \psi]\rangle$ and $\Delta\left\langle\left[\alpha_{s} G^{2}\right]\right\rangle$ should behave smoothly across the critical line. The most serious difference is that, in these channels, we cannot argue on physical grounds that the corresponding susceptibility diverges. Nonetheless, there exist lattice simulations [26] showing that the so-called quark number susceptibility rapidly increases in the transition. Thus it is plausible that also in the vector channel the SF has a vanishing threshold, and accumulates strength just above it. A similar argument in the context of the Nambu-Jona-Lasinio model can be found in Ref. [27].

\section{SUMMARY AND OUTLOOK}

We used the operator product expansion and the renormalization group equation to derive exact sum rules at finite $T$ and $\mu$ valid for asymptotically free theories. Our derivation explicitly show that logarithmic corrections cannot be neglected. We found that, depending on the theory and on the current under study, the zeroth moment of a spectral function is either independent of $T$ and $\mu$, or its change is related to the corresponding changes of the condensates of operators of lowest dimension.

In particular the zeroth moment of the scalar and pseudoscalar mesonic currents in QCD are independent of $T$ and $\mu$. As a consequence any change in the strength of the pion pole must be exactly compensated by a change in the continuum contribution. We also infer that spectral functions in the scalar and pseudoscalar channel should dramatically change near phase transition. We also find that the zeroth moment of the vector and axial-vector mesonic currents in QCD changes with $T$ and $\mu$, and these changes are related to the corresponding changes of the condensates.

Due to their generality, these exact sum rules strongly constrain the qualitative shape of SF's, in particular, near phase transitions. Since it appears that a strong deformation of the spectral function from its counter part at $(T, \mu)=0$ and perturbative cases is the most 
likely scenario near phase transitions, the information carried by these exact sum rules is particularly welcome. We urge whoever parameterizes a spectral function, e.g. in QCD sum rule type of calculations or to interpret lattice simulation data, to incorporate these exact constraints.

We have also illustrated in great detail the derivation of the sum rules, and confirmed their validity, in the Gross-Neveu model, where we can also calculate the exact spectral function. Even if this model calculation is meant mostly as an illustration of many delicate issues of the derivation (such as convergence, asymptotic expansion, Mellin transform, high momentum expansion vs. high temperature expansion, etc.), it is nevertheless comforting to find that all our general expectations about the spectral function are explicitly verified in the model.

In the future, we plan to generalize these results to baryonic currents and to analyze the phenomenological consequences of these sum rules in greater detail.

This work is supported in part by funds provided by the U.S. Department of Energy (D.O.E.) under contracts \#DE-AC02-76ER03069 and \#DE-FG06-88ER40427. 


\section{REFERENCES}

* E-mail: shuang@pierre.mit.edu and lissia@pierre.mit.edu

$\dagger \quad$ Present address.

[1] M. A. Shifman, A. I. Vainshtein and V. I. Zakharov, Nucl. Phys. B147, 385 (1979); ibid. 448; ibid. 519.

[2] For a review, see for example: S. Narison, QCD spectral sum rules (World Scientific, Singapore, 1989).

[3] M.-C. Chu, J. M. Grandy, S. Huang and J. W. Negele, Phys. Rev. D 48, 3340 (1993).

[4] For a recent review, see E. Shuryak, Rev. Mod. Phys. 61 (1993) 1.

[5] T. Hatsuda, Y. Koike and S. Lee, Nucl. Phys. B394 (1993) 221.

[6] S. Huang, Phys. Rev. D 47, 653 (1993).

[7] A. I. Bochkarev and E. Shaposhnikov, Nucl. Phys. B268 (1986) 220.

[8] H. G. Dosch and S. Narison, Phys. Lett. B203 (1988) 155.

[9] T. Hashimoto, A. Nakamura and I. O. Stamatescu, Nucl. Phys. B400, 267 (1993).

[10] G. Boyd, F. Karsch and S. Gupta, Nucl. Phys. B385, 481 (1992).

[11] M. Chu, S. Huang and S. Schramm, unpublished.

[12] J. W. Negele and H. Orland, "Quantum Many-Particle Systems", (Addision-Wesley, Reading, PA, 1988).

[13] L. S. Brown, "Quantum Field Theory", Cambridge Univ. Press, (1992).

[14] L. S. Brown, L. W. Yaffe and C. Zhai, Phys. Rev. D 46 (1992) 4712.

[15] N. Bleistein and R. A. Handelsman, "Asymptotic Expansions of Integrals", Dover Publications, Inc., New York (1986).

[16] S. Weinberg, Phys. Rev. Lett. 18, 507 (1967).

[17] For example, a recent paper appears to overlook points (3) and (4): J. I. Kapusta and E. V. Shuryak, Phys. Rev. D 46, 4694 (1994).

[18] D. Gross and A. Neveu, Phys. Rev. D 10, 3235 (1974).

[19] U. Wolff, Phys. Lett. B157, 303 (1985).

[20] A. Barducci et al., hep-th-9406117 (1994).

[21] R. F. Dashen, S. K. Ma and R. Rajaraman, Phys. Rev. D 11, 1499 (1975).

[22] S. Huang and B. Schreiber, Nucl. Phys. B426 [FS] (1994) 644.

[23] G. 't Hooft, in "The Whys of Subnuclear Physics", Ed. A. Zichichi, Plenum Press, NY (1979) 943;

A. H. Mueller, Nucl. Phys. B250, 327 (1985).

[24] T. Hatsuda and T. Kunihiro, Phys. Rev. Lett. 55, 158 (1985).

[25] F. Brown et al., Phys. Rev. Lett. 65, 2491 (1990).

[26] S. Gottlieb et al. Phys. Rev. D 38, 2888 (1988).

R. V. Gavai, J. Potvin and S. Sanielevici, Phys. Rev. D 40, 2743 (1989).

[27] T. Kunihiro, Phys. Lett. B271, 395 (1991). 


\section{FIGURES}

FIG. 1. Phase diagram of the Gross-Neveu model in $1+1$ dimension. The continuum line represent the second order phase boundary in the $\left(T / T_{c}, \mu / M\right)$-plane, while the dashed line is the first order phase boundary. The "tricritical point", where first and second order transitions merge, is shown as a heavy dot. The dotted line separates the values of $(T, \mu)$ for which the symmetry is always broken from those values of $(T, \mu)$ for which a "metastable symmetric phase" is possible. Dots show those values of $(T, \mu)$ at which we plot the spectral function in Fig. 2 and Fig. 3. Their coordinates are: $a=(0.352,0.4), b=(0.848,0.4), c=(0.875,0.4), d=(1.763,0.4), e=(1.175,0.4)$, $f=(1.763,0.4), g=(0.353,0.63), h=(0.353,0.65)$ and $k=(0.353,0.85)$.

FIG. 2. Spectral function of the $1+1$ dimensional Gross-Neveu model in pseudoscalar channel. We show several values of $(T, \mu)$ that go from the broken phase to the symmetric phase passing through the second order phase transition. The labels a, b, c, d, e and $\mathrm{f}$ correspond to the labels on the points in the $\left(T / T_{c}, \mu / M\right)$-plane shown in Fig. 1. The upper panel shows spectral functions in the broken phase. The pion poles are indicated by arrows, and are slightly displaced from their true position at $\omega=0$ for vision convenience. The length of arrows represents the magnitude of the corresponding residues. The lower panel shows spectral functions in the symmetric phase, where there is no pion pole.

FIG. 3. The notations are the same as in Fig. 2, except that now $(T, \mu)$ goes through the first order phase boundary. The coordinates of the labels $\mathrm{a}, \mathrm{g}, \mathrm{h}$ and $\mathrm{k}$ are given in Fig. 1 .

FIG. 4. Feynman diagrams for calculating the anomalous dimensions of (a) the composite operator $J_{5}$, i.e. $\gamma_{J_{5}},(\mathrm{~b})$ and of the composite operator $O_{4}$, i.e. $\gamma_{O_{4}}$.

FIG. 5. Feynman diagrams for calculating Wilson coefficients: (a) $c_{O_{4}}$ and (b) $c_{\theta_{00}}$.

FIG. 6. Feynman diagrams contributing to the vector correlator.

FIG. 7. (a) the dynamical quark mass and (b) the baryon number susceptibility as functions of temperature. 
This figure "fig1-1.png" is available in "png" format from: http://arxiv.org/ps/hep-ph/9412246v1 
This figure "fig2-1.png" is available in "png" format from: http://arxiv.org/ps/hep-ph/9412246v1 
$D$
$\sqcup$
$\frac{0}{\complement}$
$\frac{1}{2}$
0
$\longmapsto$

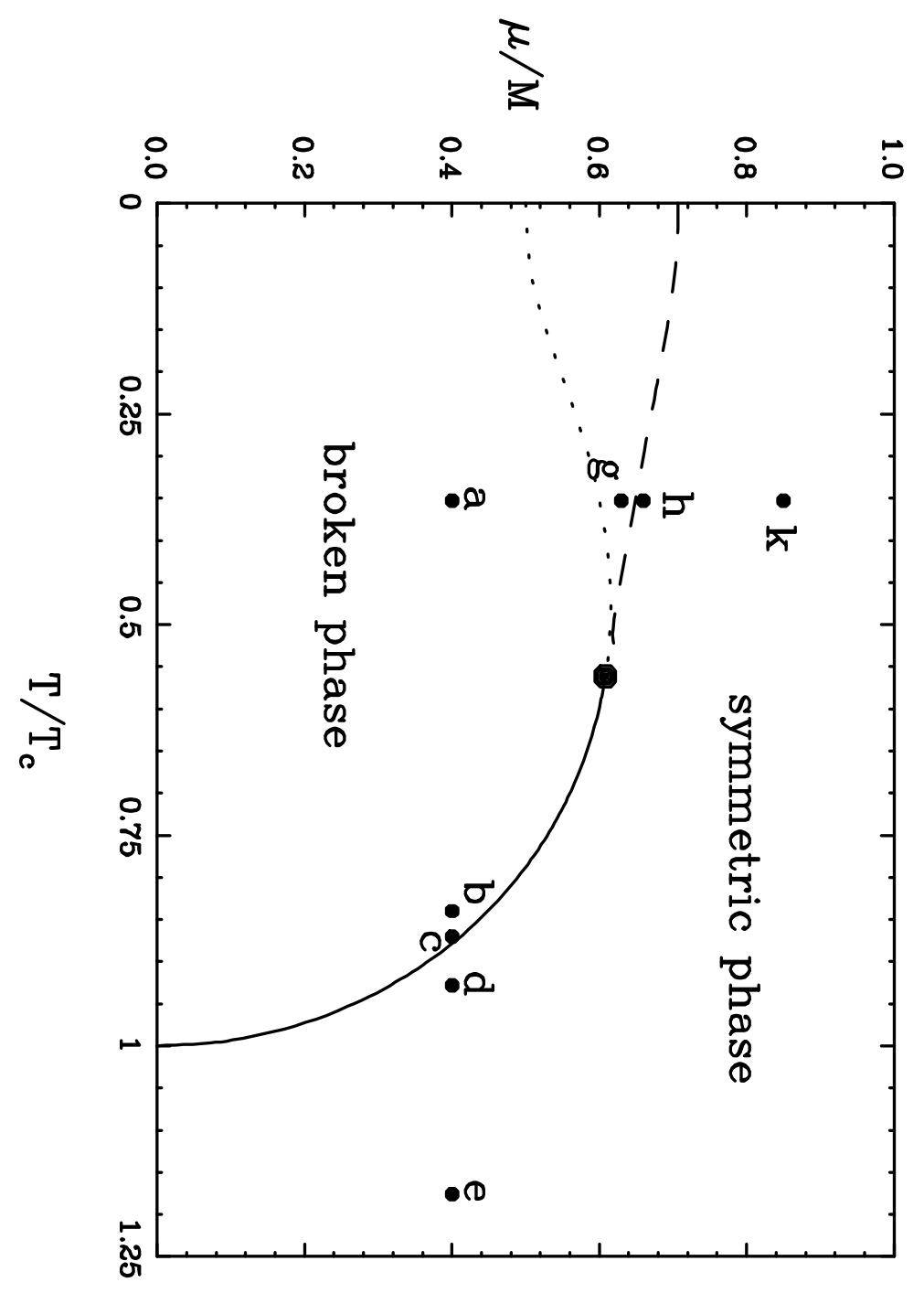


This figure "fig1-2.png" is available in "png" format from: http://arxiv.org/ps/hep-ph/9412246v1 
This figure "fig2-2.png" is available in "png" format from: http://arxiv.org/ps/hep-ph/9412246v1 

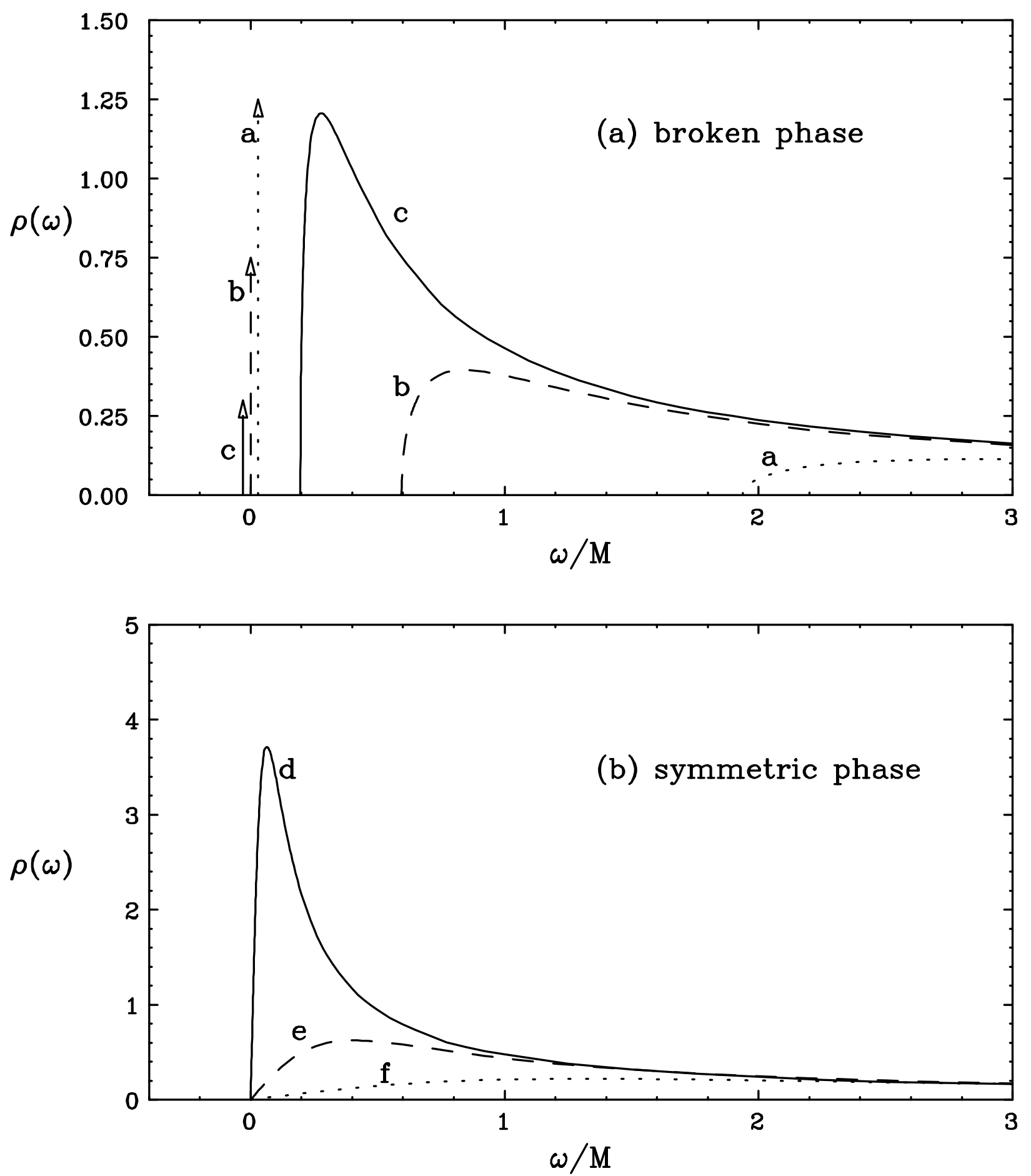

figure 2 
This figure "fig1-3.png" is available in "png" format from: http://arxiv.org/ps/hep-ph/9412246v1 

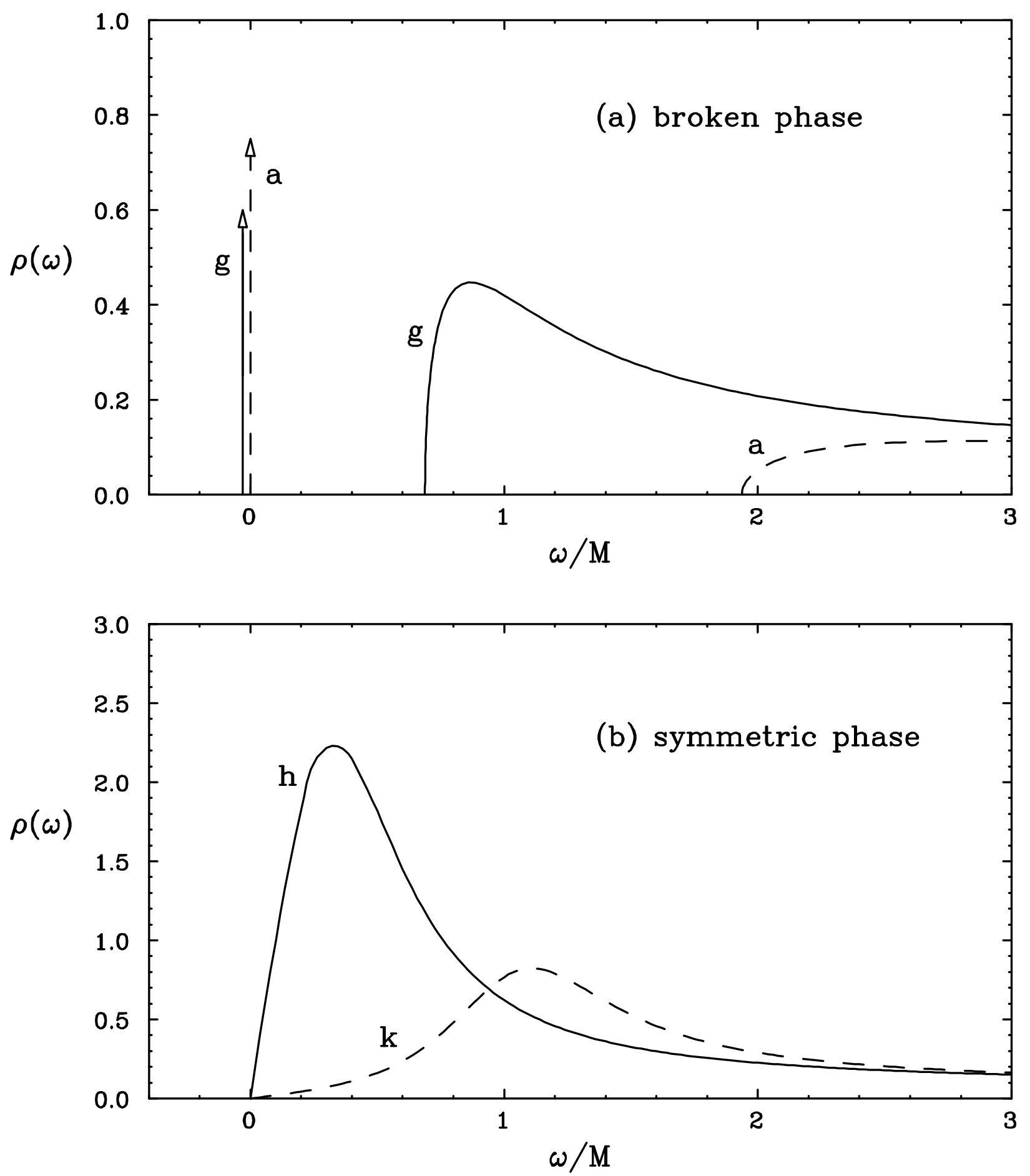

Figure 3 
This figure "fig1-4.png" is available in "png" format from: http://arxiv.org/ps/hep-ph/9412246v1 
$D$
$\stackrel{\bullet}{C}$
$\stackrel{0}{D}$
$D$
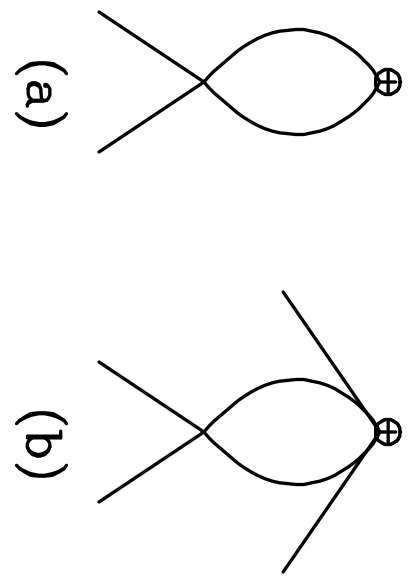
This figure "fig1-5.png" is available in "png" format from: http://arxiv.org/ps/hep-ph/9412246v1 
$\stackrel{T}{\bullet}$
$\stackrel{0}{\complement}$
$\frac{0}{0}$
0
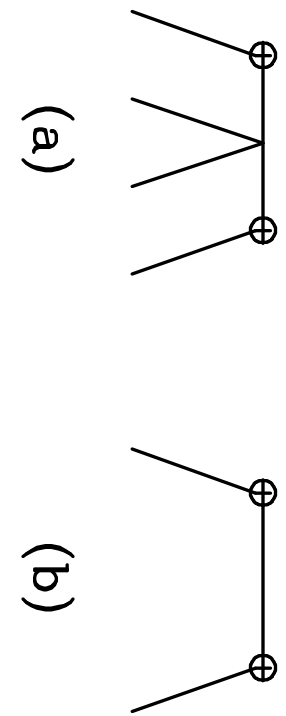
7
$\frac{1}{c}$
$\frac{0}{7}$
0
0

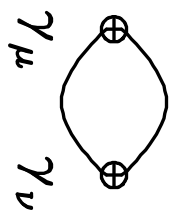

$+$

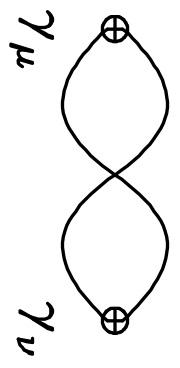

$+$

$\vdots$ 

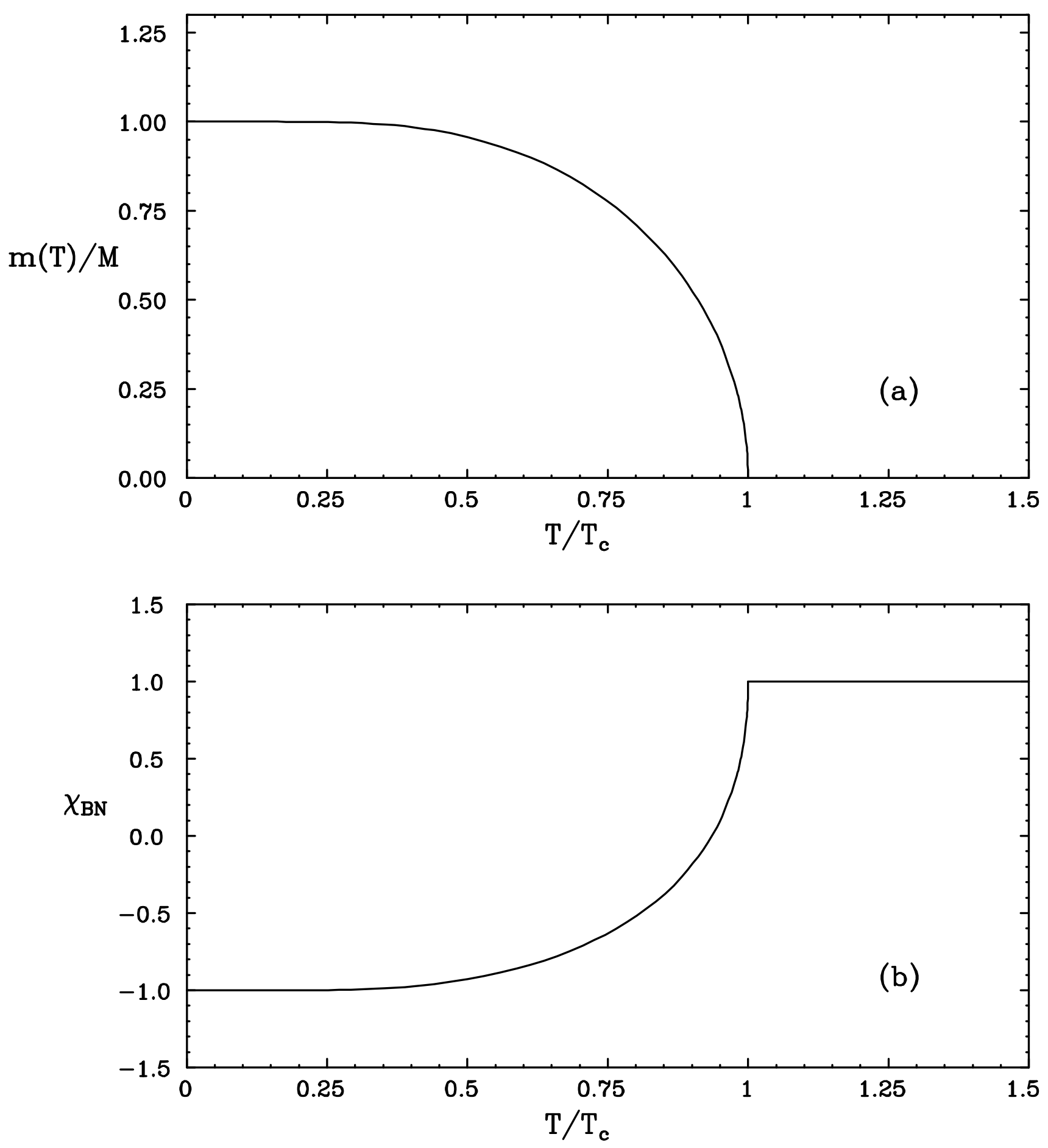

Figure 7 